\title{
The Downward Influence of Uncertainty in the Northern Hemisphere Stratospheric Polar Vortex Response to Climate Change
}

\author{
ISLA R SIMPSON \\ Climate and Global Dynamics Laboratory, National Center for Atmospheric Research, Boulder, Colorado \\ PETER HitCHCOCK \\ Laboratoire de Météorologie Dynamique, École Polytechnique, Palaiseau, France \\ RICHARD SEAGER AND YUTIAN WU \\ Lamont-Doherty Earth Observatory, Columbia University, New York, New York \\ PATRICK CALLAGHAN \\ Climate and Global Dynamics Laboratory, National Center for Atmospheric Research, Boulder, Colorado
}

(Manuscript received 23 January 2018, in final form 3 May 2018)

\begin{abstract}
General circulation models display a wide range of future predicted changes in the Northern Hemisphere winter stratospheric polar vortex. The downward influence of this stratospheric uncertainty on the troposphere has previously been inferred from regression analyses across models and is thought to contribute to model spread in tropospheric circulation change. Here we complement such regression analyses with idealized experiments using one model where different changes in the zonal-mean stratospheric polar vortex are artificially imposed to mimic the extreme ends of polar vortex change simulated by models from phase 5 of the Coupled Model Intercomparison Project (CMIP5). The influence of the stratospheric vortex change on the tropospheric circulation in these experiments is quantitatively in agreement with the inferred downward influence from across-model regressions, indicating that such regressions depict a true downward influence of stratospheric vortex change on the troposphere below. With a relative weakening of the polar vortex comes a relative increase in Arctic sea level pressure (SLP), a decrease in zonal wind over the North Atlantic, drying over northern Europe, and wetting over southern Europe. The contribution of stratospheric vortex change to intermodel spread in these quantities is assessed in the CMIP5 models. The spread, as given by 4 times the across-model standard deviation, is reduced by roughly $10 \%$ on regressing out the contribution from stratospheric vortex change, while the difference between models on extreme ends of the distribution in terms of their stratospheric vortex change can reach up to $50 \%$ of the overall model spread for Arctic SLP and $20 \%$ of the overall spread in European precipitation.
\end{abstract}

\section{Introduction}

As the planet continues to warm under rising greenhouse gas (GHG) concentrations, we strive for an improved assessment and understanding of how the large-scale atmospheric circulation and associated regional climate is expected to change in the future. While general circulation models (GCMs), in general, simulate a poleward shifting of the zonal-mean midlatitude westerlies and associated storm tracks as the planet

Corresponding author: Isla R. Simpson, islas@ucar.edu warms (Yin 2005; Kidston and Gerber 2010; Swart and Fyfe 2012; Wilcox et al. 2012; Barnes and Polvani 2013; Chang et al. 2012), there is considerable spread among models in the magnitude of this response (Harvey et al. 2012; Woollings and Blackburn 2012; Delcambre et al. 2013). Regionally, changes to the large-scale stationary waves lead to deviations from this zonal-mean poleward-shifting view (Stephenson and Held 1993; Joseph et al. 2004; Simpson et al. 2014) and enhanced uncertainty resulting from the varied model representation of stationary waves and their climate change response. 
Some of this uncertainty in the climate change response will be irreducible (Hawkins and Sutton 2009), arising from internal variability of the climate system. The remaining uncertainty, under a consistent forcing scenario, arises from structural differences among models in how they represent the processes of relevance for the large-scale circulation. It is this uncertainty that we can hope to reduce, through improved understanding and representation of the relevant processes.

The shifting of the midlatitude westerlies is thought to arise primarily as a response to altered temperature gradients produced via the thermodynamic effects of increasing GHG concentrations [see the review paper by Shaw et al. (2016) and references therein]. The warming of the tropical upper troposphere and the cooling of the stratosphere are each thought to shift the westerlies poleward while this is partially offset, in the Northern Hemisphere ( $\mathrm{NH})$, by the influence of amplified Arctic warming during boreal winter (Lorenz and DeWeaver 2007; Butler et al. 2010; Harvey et al. 2014). Model differences in the representation of the various feedback processes that modify the large-scale temperature gradients, such as cloud and water vapor radiative effects (Voigt and Shaw 2015; Ceppi and Hartmann 2016) or sea ice loss and albedo changes (Barnes and Screen 2015), likely contribute substantial uncertainty (Harvey et al. 2014; Wenzel et al. 2016; Ceppi and Shepherd 2017). In addition, the varied model representation of the mean-state circulation may give rise to differences in the climate change response through modification of the dynamics of eddy-mean flow feedbacks or other processes (Kidston and Gerber 2010; Barnes and Hartmann 2010; Sigmond and Scinocca 2010; Simpson and Polvani 2016).

In the $\mathrm{NH}$ wintertime, a potential source of uncertainty in tropospheric circulation change is the representation of the stratosphere and the downward influence of stratospheric circulation changes, as models do not agree on how the vortex will change in the future (Manzini et al. 2014; Butchart et al. 2010). Earlier studies on this topic suggested a dependence of the stratospheric response on vertical resolution with resulting tropospheric impacts but did not agree on the sign of this influence (Shindell et al. 1999; Scaife et al. 2012; Karpechko and Manzini 2012), while Gillett et al. (2002) found no significant influence of vertical resolution on the stratospheric response to climate change. As the number of high-top models available within multimodel intercomparisons has increased (Gerber et al. 2012; Charlton-Perez et al. 2013), it has become clear that there is actually no consistent link between a model's top or stratospheric resolution and how it responds to increasing GHGs (Butchart et al. 2010;
Manzini et al. 2014). The processes that give rise to strengthening, weakening, or no change in the $\mathrm{NH}$ wintertime polar vortex remain an open question. Past studies have argued that the way in which a model's stratospheric circulation is tuned in the presence of parameterized gravity waves can impact future changes in stratospheric wave propagation (Sigmond et al. 2008), while others have shown a link between a model's vortex response and the altered source of stratospheric planetary waves from the troposphere below (Karpechko and Manzini 2017).

Our focus here is not on what gives rise to model diversity in the stratospheric circulation response to rising GHGs, but rather, what is the downward influence of this diversity on the troposphere below? Our modeling study is heavily influenced by the analysis based on phase 5 of the Coupled Model Intercomparison Project (CMIP5) of Manzini et al. (2014, hereinafter M2014). They presented regressions, across models, of tropospheric circulation change onto a measure of polar vortex change under the representative concentration pathway 8.5 (RCP8.5) forcing scenario and showed that models that exhibit a greater weakening of the stratospheric polar vortex in the future exhibit less of a reduction in Arctic sea level pressure (SLP) and a reduced poleward shifting of the tropospheric westerlies. A problem with such analyses of multimodel ensembles is that the diagnosed influence may in fact be caused by a multitude of other factors, such as differences in model resolution, tuning, representation of physical processes, and so on, in addition to the phenomenon of interest. In addition, while stratospheric circulation variability has an influence on the troposphere below (e.g., Baldwin and Dunkerton 2001), the primary driver of stratospheric variability in the first place is variations in the wave activity propagating upward from the troposphere. So, identified links between aspects of the stratospheric and tropospheric circulation could represent a causal connection in either direction. While some attempt at establishing cause and effect has been made through lagged regressions (M2014), idealized experiments, designed to unambiguously test and quantify the influence of stratospheric circulation changes on the troposphere below, are needed. This is what we provide here.

The methodology we use is to nudge the stratospheric zonal-mean climatological circulation, within one model, to states that span the range of CMIP5 projections of the zonal-mean stratospheric polar vortex under the RCP8.5 scenario. In this way, we can assess the influence of changes in the zonal mean, climatological, stratospheric boundary conditions on the troposphere below. We stress that these experiments cannot tell us everything about the potential role the stratosphere may play 
in tropospheric circulation change. What they can tell us is the climatological circulation changes that are produced in response to climatological zonal-mean changes in the stratosphere. For example, the mean meridional circulation produced as a "downward control" (Haynes et al. 1991) response to the altered climatological stratospheric forcings that drive the vortex changes should be represented in this framework (Hitchcock and Haynes 2014) along with mechanisms whereby changes in the lower-stratospheric state affect tropospheric transient eddies or larger-scale planetary waves, with ensuing impacts on the tropospheric zonal-mean circulation (Song and Robinson 2004; Kushner and Polvani 2004; Wittman et al. 2007; Lorenz and DeWeaver 2007; Simpson et al. 2009). What these experiments cannot tell us is the influence of nonlinear effects associated with largeamplitude events. For example, if nonlinearities associated with a change in the number of sudden stratospheric warmings (SSWs) or with planetary wave reflection from anomalous polar vortex configurations (Perlwitz and Harnik 2003; Shaw et al. 2010) were important to the time-averaged response, the present approach of relaxing the zonal mean toward a climatological mean state would not capture such effects. Nevertheless, it will be shown that the experiments demonstrate a similar stratospheric influence on climatological tropospheric circulation change to that inferred from the regression analysis of M2014, suggesting that the mechanisms that can be captured with this methodology dominate in the stratosphere's role in intermodel spread of future wintertime climatological change.

We begin with an initial analysis of the CMIP5 intermodel spread in future predicted changes in the $\mathrm{NH}$ wintertime stratospheric polar vortex in section 2 . This motivates the design of the model experiments, which will be described, along with our CMIP5 analysis methodology, in section 3. Results are presented in section 4, followed by discussion and conclusions in section 5 .

\section{The intermodel spread in $\mathrm{NH}$ winter stratospheric circulation change}

The motivation for these experiments is the complete lack of agreement among CMIP5 models on the future of the NH winter zonal-mean stratospheric polar vortex under a rising GHG scenario. While M2014 already discussed this wide spread among models, we illustrate this here again in Fig. 1 for the set of CMIP5 models that we use to design the perturbation experiments. This makes use of the 35 models and ensemble members listed in Table 1 under the historical and RCP8.5 forcing scenarios (Taylor et al. 2012) with the ensemble mean for each model calculated prior to the multimodel mean.
The "past" is the 27-yr period between 1979 and 2005 of the historical simulations, and "future" is the $30-y r$ period between 2070 and 2099 of the RCP8.5 simulations.

The multimodel-mean future - past difference in December-February (DJF)-averaged zonal-mean zonal wind $\bar{u}$ is shown in Fig. 1b. In the region of the stratospheric polar vortex, for example, poleward of about $60^{\circ} \mathrm{N}$ at $10 \mathrm{hPa}$, the predicted change in zonal-mean zonal wind is very small but the standard deviation of the multimodel-mean response (Fig. 1c) is considerably larger than the ensemble-mean response itself, which speaks to the lack of agreement among models on the sign of the zonal wind change here. This large variation among models in the stratospheric polar vortex region remains after accounting for the model spread in climate sensitivities by first regressing out the contribution that is linearly related to the globally averaged surface temperature change $T_{s}$ (Fig. 1d); that is, here we assume the change in $\bar{u}$ is given by

$$
\bar{u}=b T_{s}+\bar{u}^{\prime},
$$

with the constant $b$ determined by linear regression, and we examine the standard deviation of $\bar{u}^{\prime}$.

This wide spread among models is further illustrated in Figs. 1e and 1f, which show the future - past difference in $\bar{u}$ at $10 \mathrm{hPa}$ averaged between $60^{\circ}$ and $75^{\circ} \mathrm{N}$ (area weighted) for each model individually, both before and after regressing out the contribution related to the globally averaged $T_{s}$ change, with the latter leading to only minor alterations in the ordering of models and the magnitude of their response. Considering the response in Fig. 1e, the models can be roughly equally divided by the sign of their response with 17 models exhibiting a decrease in the zonal-mean zonal wind and 18 exhibiting an increase.

Next, we assess whether the change in zonal-mean zonal wind for an individual model is significant when compared with an equivalent sampling of the preindustrial control simulation for that model. Consider CESM1(WACCM), which has seven past samples and three future samples, as an example. Seven chunks of length $27 \mathrm{yr}$ and three chunks of length $30 \mathrm{yr}$ are sampled at random from the preindustrial control simulation (these chunks may overlap) to represent the seven past samples and three future samples, respectively. The mean climatology for each chunk is calculated followed by the mean over the seven chunks that mimic the past and the three chunks that mimic the future. The difference between these means is then calculated, and this represents one sample of the difference that could be obtained between three 30-yr climatologies and seven 27-yr climatologies 
(a) Past, DJF

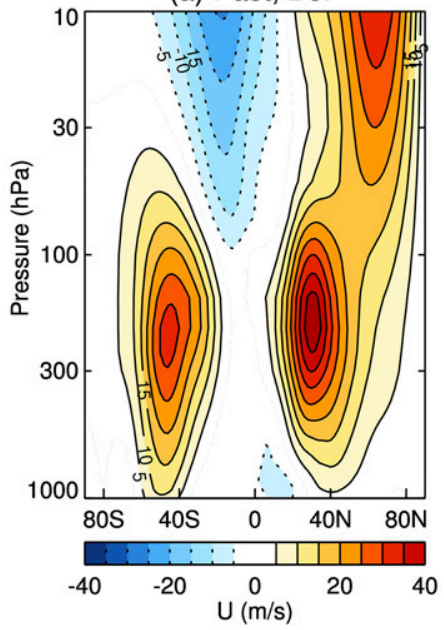

(b) Future-Past, DJF

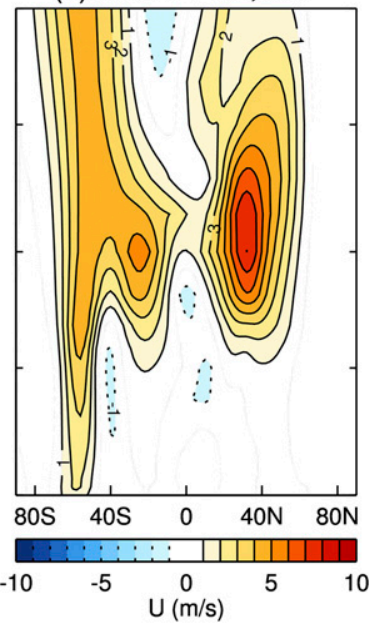

(e) DJF U at $10 \mathrm{hPa}, 60 \mathrm{~N}-75 \mathrm{~N}$
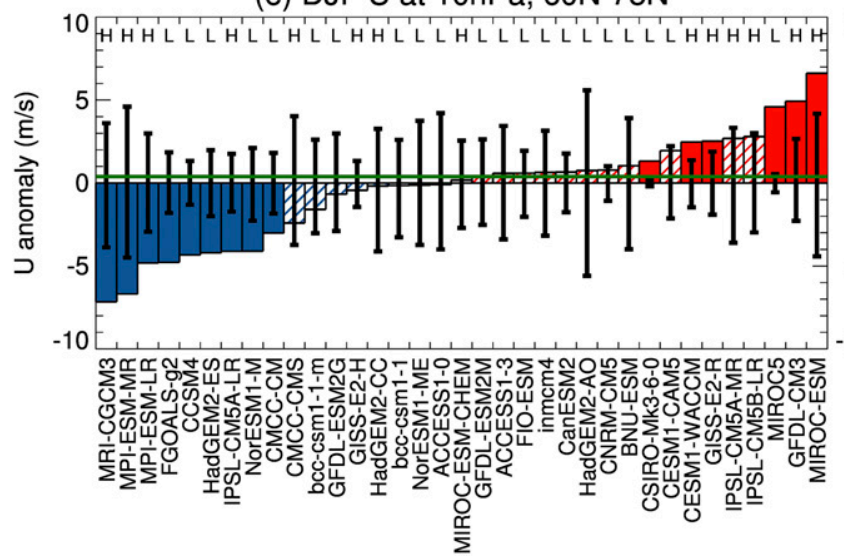

(c) $\sigma$ of (b)

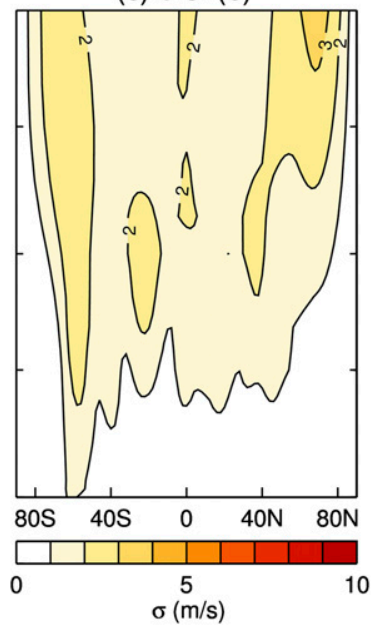

(d) As (c) without $T_{\mathrm{s}}$

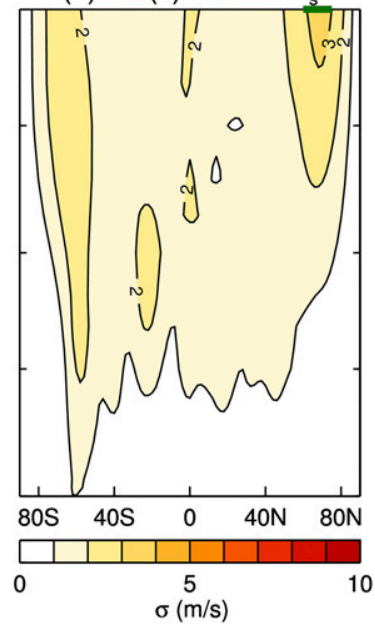

(f) As (e) but after regressing out $\mathrm{T}_{\mathrm{s}}$ contribution
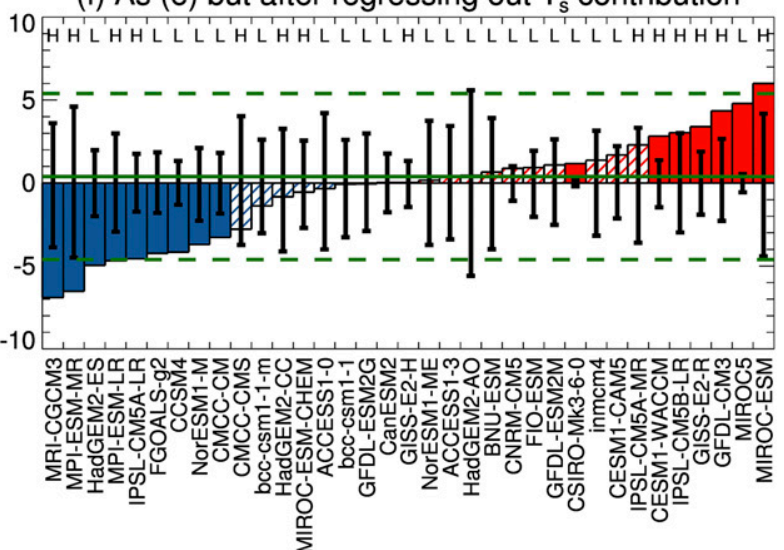

FIG. 1. DJF zonal-mean zonal wind: multimodel-mean (a) past climatology and (b) future - past difference. (c) The across-model $\sigma$ of the future - past difference. (d) As in (c), but after regressing out the component of the zonal wind difference that is linearly related to the globally averaged surface temperature increase. (e) The future - past difference at $10 \mathrm{hPa}$ averaged from $60^{\circ}$ to $75^{\circ} \mathrm{N}$. Models are shown in order of increasing zonal wind difference. Black bars show the 2.5th-97.5th percentile range of bootstrap sample differences from a model's preindustrial control simulation (see section 2), and solid/hatched bars depict anomalies that are/are not significantly different from zero at the $5 \%$ level. The letters $\mathrm{H}$ and $\mathrm{L}$ depict whether a model is considered high or low top, determined by whether or not the model lid is above $1 \mathrm{hPa}$ (Charlton-Perez et al. 2013). (f) As in (e), but after removing the component that is linearly related to the globally averaged surface temperature increase. The solid green lines in (e) and (f) show the zonal wind anomaly for CESML46 FREE4x FREE1x, and the dashed lines in (f) show CESML46 FREE4x - FREE1 $\pm 5 \mathrm{~m} \mathrm{~s}^{-1}$ (i.e., the magnitude of $\bar{u}_{\mathrm{pv}}$ anomalies used to define the perturbations for STRONG4x and WEAK4x).

when sampled from that model's control simulation. This is then repeated 5000 times to build up a distribution of zonal wind anomalies that could be obtained with a sample of this size. This procedure is then followed for each model with the sample size that is equivalent to the number of past and future members used (Table 1). The black bars in Figs. 1e and $1 \mathrm{f}$ show the 2.5th-97.5th percentile range of these preindustrial control samples, and note that intermodel variations in this range can arise from both differences in the sample size and differences in stratospheric variability between the models. For a model where the zonal wind response lies outside of this range we can conclude that there is less than a $2.5 \%$ chance of obtaining an anomaly that big (or that small) from sampling alone (equivalent to the response being significantly different from zero at the $5 \%$ level by a two-sided test). This assumes that the stratospheric variability does not change substantially from the preindustrial to the historical or RCP8.5 climates, which is reasonable given the lack of consensus on this point (Rind et al. 1998; McLandress and Shepherd 2009; Bell et al. 2010; Karpechko and Manzini 2012; Mitchell et al. 2012; Ayarzagüena et al. 2018). 
TABLE 1. List of models and historical and RCP8.5 members used in the CMIP5 analysis. The subset of eight models used to define the stratospheric perturbation above $10 \mathrm{hPa}$ are highlighted in boldface. (Expansions of acronyms are available online at http:// www.ametsoc.org/PubsAcronymList.)

\begin{tabular}{|c|c|c|}
\hline Model & $\begin{array}{c}\text { Historical } \\
\text { members }\end{array}$ & $\begin{array}{l}\text { RCP } 8.5 \\
\text { members }\end{array}$ \\
\hline ACCESS1.0 & 3 & 1 \\
\hline ACCESS1.3 & 3 & 1 \\
\hline BCC_CSM1.1 & 3 & 1 \\
\hline BCC_CSM1.1(m) & 3 & 1 \\
\hline BNU-ESM & 1 & 1 \\
\hline CanESM2 & 5 & 5 \\
\hline CCSM4 & 6 & 6 \\
\hline CESM1(CAM5) & 3 & 3 \\
\hline CESM1(WACCM) & 7 & 3 \\
\hline CMCC-CM & 1 & 1 \\
\hline CMCC-CMS & 1 & 1 \\
\hline CNRM-CM5 & 10 & 5 \\
\hline CSIRO Mk3.6.0 & 10 & 10 \\
\hline FGOALS-g2 & 5 & 1 \\
\hline FIO-ESM & 3 & 3 \\
\hline GFDL CM3 & 5 & 1 \\
\hline GFDL-ESM2G & 1 & 1 \\
\hline GFDL-ESM2M & 1 & 1 \\
\hline GISS-E2-H & 5 & 2 \\
\hline GISS-E2-R & 6 & 2 \\
\hline HadGEM2-AO & 1 & 1 \\
\hline HadGEM2-CC & 3 & 1 \\
\hline HadGEM2-ES & 5 & 3 \\
\hline INM-CM4.0 & 1 & 1 \\
\hline IPSL-CM5A-LR & 6 & 4 \\
\hline IPSL-CM5A-MR & 3 & 1 \\
\hline IPSL-CM5B-LR & 1 & 1 \\
\hline MIROC5 & 5 & 3 \\
\hline MIROC-ESM & 3 & 1 \\
\hline MIROC-ESM-CHEM & 1 & 1 \\
\hline MPI-ESM-LR & 3 & 3 \\
\hline MPI-ESM-MR & 3 & 1 \\
\hline MRI-CGCM3 & 3 & 1 \\
\hline NorESM1-M & 3 & 1 \\
\hline NorESM1-ME & 1 & 1 \\
\hline
\end{tabular}

This analysis then reveals that, at $10 \mathrm{hPa}$ and $60^{\circ}-75^{\circ} \mathrm{N}$, nine models exhibit a significant weakening of the zonalmean zonal wind and six [seven if the globally averaged $T_{s}$ contribution is first regressed out (Fig. 1f)] exhibit a significant strengthening, while 20 models exhibit a response that is not larger than expected from the sampling of internal variability (Fig. 1e). As in M2014, there is no clear link between a model's lid height and the response (the letters $\mathrm{H}$ and $\mathrm{L}$ in Figs. 1e,f).

While our results are similar to M2014, the multimodelmean weakening of the high-latitude winds is slightly reduced in our group of models (note the lack of a $-1 \mathrm{~m} \mathrm{~s}^{-1}$ contour in Fig. 1b compared to Fig. 2a in M2014, where the ensemble-mean response surpasses $-1 \mathrm{~m} \mathrm{~s}^{-1}$ ). In addition, M2014 concluded that around 70\% of models in CMIP5 exhibit a weakening of the polar vortex, while here we find it is roughly $50 \%$. This is likely partly due to the increased number of models included here (35 compared to $24)$ and is also partly due to the different latitude region considered $\left(60^{\circ}-75^{\circ} \mathrm{N}\right.$ here, compared to $70^{\circ}-80^{\circ} \mathrm{N}$ in M2014). Three of the significantly strengthening models in Fig. 1e (and four in Fig. 1f) were not included in the analysis of $\mathrm{M} 2014$, and if we use the $70^{\circ}-80^{\circ} \mathrm{N}$ measure for the current set of models, we find $60 \%$ of the models exhibit a weakening.

In summary, there is no clear consensus on the response of the stratospheric polar vortex to increasing GHGs over the coming century. Models predict that the vortex may significantly strengthen, significantly weaken, or exhibit no significant change. This motivates the following model experiments, which aim to assess to what extent this model spread, in future changes in the strength of the stratospheric zonal-mean polar vortex, may impact the troposphere below.

\section{Model experiments and CMIP5 analysis}

\section{a. The model}

The experiments are performed using a modified version of the Community Earth System Model (CESM), version 1.2, which consists of the Community Atmosphere Model, version 5 (CAM5), coupled to the Parallel Ocean Program model, version 2 (POP2), and the Community Land Model, version 4 (CLM4). The atmosphere model uses the finite-volume dynamical core at approximately $0.9^{\circ} \times 1.25^{\circ}$ latitude-longitude resolution but, in contrast to the default configuration of CAM5 with 30 levels and a model top at $2 \mathrm{hPa}$, we use a 46-level configuration that extends to $0.3 \mathrm{hPa}$, described in Richter et al. (2015). This version also contains the nonorographic gravity wave drag parameterization described in Richter et al. (2010), which results in the free-running model exhibiting an internally generated quasi-biennial oscillation along with reasonable SSW statistics. A similar version, but making use of the spectral-element dynamical core with prescribed sea surface temperatures, has been previously used in the studies of Richter et al. (2015) and Polvani et al. (2017). We will refer to the model configuration used here as CESML46.

\section{b. Simulations}

The model simulations are summarized in Table 2. These consist of a 260-yr-long free-running control simulation (FREE1x) with GHG concentrations specified at preindustrial levels (284.7 ppm), along with a 272yr-long $4 \times \mathrm{CO}_{2}$ simulation (FREE4x) in which carbon dioxide $\left(\mathrm{CO}_{2}\right)$ concentrations are elevated to 4 times 
TABLE 2. A description of the CESML46 model experiments.

\begin{tabular}{lcl}
\hline \multicolumn{1}{c}{ Name } & Length $(\mathrm{yr})$ & \multicolumn{1}{c}{ Description } \\
\hline FREE1x & 260 & Free-running preindustrial control. \\
FREE4x & 272 & Free-running with abrupt quadrupling of $\mathrm{CO}_{2}$. \\
NUDG1x & 222 & Nudged preindustrial control. \\
NUDG4x & 222 & Nudged quadrupling of $\mathrm{CO}_{2}$ initialized from year 50 of FREE4x. \\
STRONG4x & 222 & As in NUDG4x, but nudged to have a strengthening of the polar vortex. \\
WEAK4x & 222 & As in NUDG4x, but nudged to have a weakening of the polar vortex. \\
\hline
\end{tabular}

preindustrial levels (1138.8 $\mathrm{ppm})$ from the beginning of the simulation.

The remaining four experiments employ a relaxation/ nudging of the zonal-mean state of the stratosphere to various target climatologies following a similar methodology to Simpson et al. (2011) and Hitchcock and Simpson (2014). For a given field $X$, with zonal-mean component $\bar{X}$, an additional relaxation of the form $-K(p)\left(\bar{X}-\bar{X}_{o}\right) / \tau_{N}$ is applied to the prognostic equation for $X$ at all grid points, where $\bar{X}_{o}$ is the specified zonal-mean target state, and $\tau_{N}$ is a relaxation time scale of $6 \mathrm{~h}$. The vertical profile $K(p)$ is set to zero below $64 \mathrm{hPa}$ and increases linearly to 1 at $28 \mathrm{hPa}$ such that the model is freely running below $64 \mathrm{hPa}$ and fully constrained above $28 \mathrm{hPa}$. Nudging is performed on $\bar{u}$ and zonal-mean temperature $\bar{T}$ and meridional wind $\bar{v}$, and the target climatology is updated at 6-hourly intervals.

The first of these experiments (NUDG1x) is a preindustrial simulation in which the zonal-mean state of the stratosphere is relaxed toward the seasonally varying climatology of FREE1x (specifically, the first four harmonics of the seasonal cycle averaged over years 10 to 260 of FREE1x). The second (NUDG4x) is a $4 \times \mathrm{CO}_{2}$ simulation that is branched off from year 50 of FREE4x. Year 50 was chosen as it is after the initial rapid warming in response to elevated $\mathrm{CO}_{2}$ has slowed, with only the slower ocean adjustment occurring throughout the remainder of the simulation (Fig. 2). ${ }^{1}$ In NUDG4x, the zonal-mean state of the stratosphere is nudged toward the first four harmonics of the seasonally varying climatology from years 50 to 272 of FREE4x. This pair of simulations is, therefore, analogous to FREE1x and FREE4x, but rather than having a freely evolving

\footnotetext{
${ }^{1}$ The difference in $\mathrm{CO}_{2}$ between 2070 and 2099 of RCP8.5 and 1979-2005 of historical experiments is around $442 \mathrm{ppm}$ while the $\mathrm{CO}_{2}$ anomalies imposed in CESML46 are close to double that at $854.1 \mathrm{ppm}$. The CESML46 simulations are lacking the increase in other GHGs, but the greater increase in $\mathrm{CO}_{2}$ and the more equilibrated ocean leads to an overall greater warming than found in CMIP5; however, we do not consider the different magnitude of warming to impact the conclusions regarding the stratospheric influence, as discussed further in section 5 .
}

stratosphere, the zonal-mean stratospheric state is nudged toward the climatologies from FREE1x and FREE4x, respectively. This allows us to confirm that the relaxation does not substantially alter the tropospheric response to increased $\mathrm{CO}_{2}$.

The final two experiments, WEAK4x and STRONG4x, are also $4 \times \mathrm{CO}_{2}$ simulations branched from year 50 of FREE4x with the zonal-mean stratospheric state nudged toward the seasonally varying climatology of years 50 to 272 of FREE4x but with added perturbations that are designed to span the CMIP5 model spread in the zonalmean stratospheric polar vortex response to climate change (Fig. 1).

Each of the nudged experiments are run for 222 years so that they are sampling the same period of response to $4 \times \mathrm{CO}_{2}$ as years $50-272$ of the FREE4x simulation, which is the period used to define the nudging target state and the period used for comparison.

\section{c. Perturbation design}

We will refer to the zonal wind anomaly at $10 \mathrm{hPa}$ and $60^{\circ}-75^{\circ} \mathrm{N}$, after regressing out the component related to globally averaged $T_{s}\left[u^{\prime}\right.$ in (1); Fig. 1f] as the "polar vortex index" $\bar{u}_{\mathrm{pv}}$ in all subsequent analyses. Note that

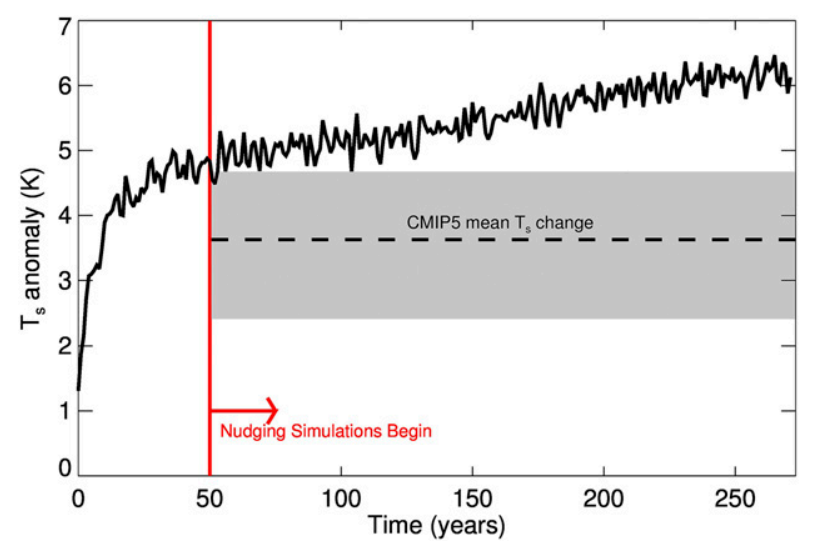

FIG. 2. (a) DJF globally averaged temperature anomaly in FREE4x relative to the FREE1x climatology. The gray-shaded region and dashed black line show the CMIP5 range and multimodel mean of the globally averaged DJF future - past difference. 
the imposed perturbation would not be substantially altered if the contribution associated with $T_{s}$ were not first regressed out. As measured by this index, the FREE4x - FREE1 $\bar{u}$ anomalies lie near the center of the CMIP5 distribution; that is, CESML46 neither exhibits a substantial strengthening nor weakening of the polar vortex under elevated $\mathrm{CO}_{2}$ (see the solid green horizontal line in Fig. 1f).

Since CESML46's $\bar{u}_{\mathrm{pv}}$ response lies close to the CMIP5 multimodel mean, we perform two experiments, WEAK $4 \mathrm{x}$ and STRONG4x, in which the $\bar{u}$ target states above $64 \mathrm{hPa}$ are given by the $4 \times \mathrm{CO}_{2}$ target state $\bar{u}_{4 \mathrm{x}}$ plus perturbations with an increase or decrease in $\bar{u}_{\mathrm{pv}}$ of $5 \mathrm{~m} \mathrm{~s}^{-1}$ and with latitude-pressure structure $\beta_{u}(\phi, p)$ and seasonality $S(t)$ derived from the CMIP5 models; that is,

$$
\begin{aligned}
& \begin{aligned}
\text { STRONG4x: } \bar{u}(\phi, p, t)= & \bar{u}_{4 \mathrm{x}}(\phi, p, t) \\
& +5 \beta_{u}(\phi, p) S(t) \text { and } \\
\text { WEAK4x: } \bar{u}(\phi, p, t)= & \bar{u}_{4 \mathrm{x}}(\phi, p, t)-5 \beta_{u}(\phi, p) S(t),
\end{aligned}
\end{aligned}
$$

with analogous expressions for $\bar{T}$ and $\bar{v}$, and $\phi, p$, and $t$ referring to latitude, pressure, and time, respectively. The latitude-pressure structure $\beta_{u}$ and seasonality $S(t)$ are the anomalies associated with an increase in $\bar{u}_{\mathrm{pv}}$ of $1 \mathrm{~m} \mathrm{~s}^{-1}$, as described in more detail in the appendix and shown in Figs. A1 and A2. The STRONG4x and WEAK $4 \mathrm{x}$ target climatologies are, therefore, designed to mimic the anomalies associated with an increase or decrease in $\bar{u}_{\mathrm{pv}}$ of $5 \mathrm{~m} \mathrm{~s}^{-1}$, that is, $\bar{u}_{\mathrm{pv}}$ anomalies that are at the edges of the CMIP5 range, roughly encompassing $90 \%$ of the models (dashed green lines in Fig. 1f). We will focus on the difference in tropospheric responses between WEAK4x and STRONG4x (i.e., we are considering the impact of a difference in $\bar{u}_{\mathrm{pv}}$ of $\Delta \bar{u}_{\mathrm{pv}}$, with $\left.\Delta \bar{u}_{\mathrm{pv}}=10 \mathrm{~m} \mathrm{~s}^{-1}\right)$.

\section{d. CMIP5 analysis}

We complement the model experiments with additional analysis of the CMIP5 model spread to assess to what extent the model experiments reproduce the stratospheric influence that would be inferred from across-model regression analysis. While this can largely be considered a confirmation of the analysis of M2014, we present it here for direct comparison with the model simulations. Even though our methods, number of models, and ensemble members, and the way in which we present the stratospheric contribution to intermodel spread, differ slightly from that of M2014, the conclusions are, reassuringly, essentially the same. The past and future periods are the same as those considered in section 2 and for a given future past difference field $X$, the regression coefficient $\beta_{X}$ of this field onto $\bar{u}_{\mathrm{pv}}$ across the 35 CMIP5 models is given by

$$
\begin{aligned}
X(\text { model })= & \alpha_{X}(\phi, p)+\beta_{X}(\phi, p) \bar{u}_{\mathrm{pv}}(\text { model }) \\
& +\varepsilon_{X}(\text { model })
\end{aligned}
$$

We will present this regression coefficient multiplied by $\Delta \bar{u}_{\mathrm{pv}}$, such that it represents the anomalies that would be associated with a difference in $\bar{u}_{\mathrm{pv}}$ of $10 \mathrm{~m} \mathrm{~s}^{-1}$, that is, equivalent to the difference between the WEAK4x and STRONG4x model experiments (Fig. 1f). Note that for all fields $X$, we first regress out the component that is linearly related to the globally averaged $T_{s}$ change, but this has no discernable influence on $\beta_{X}$ for each field shown.

\section{e. Assessing the stratospheric contribution to intermodel spread}

We also wish to assess the magnitude of the potential influence of the stratosphere relative to the total CMIP5 model spread. For this purpose, we define the CMIP5 model spread as $4 \sigma$, where $\sigma$ is the across-model standard deviation; that is, for a normal distribution, this is the range within which $95 \%$ of samples lie. We show two measures of stratospheric influence. The first, measure 1, consists of assessing, within CMIP5, the reduction in $4 \sigma$ that arises from regressing out the contribution that is linearly related to $\bar{u}_{\mathrm{pv}}$, that is, $\left[\sigma(X)-\sigma\left(\varepsilon_{X}\right)\right] / \sigma(X)$ [see (4)]. The second, measure 2 , is the magnitude of the difference between models on opposite ends of the scale in terms of their stratospheric response, expressed as a percentage of the CMIP5 spread. This could be assessed by the ratio $\beta_{X} \Delta u_{\mathrm{pv}} / 4 \sigma(X)$ or alternatively by the ratio of the difference between the WEAK4x and STRONG4x experiments to the CMIP5 $4 \sigma(X)$ range. Throughout the text we will use the difference between WEAK4x and STORNG4 $\mathrm{x}$ to make this assessment but quote the values of $\beta_{X} \Delta u_{\mathrm{pv}} / 4 \sigma(X)$ for particular regions in Table 3. Measure 1 indicates how much model spread would be reduced if uncertainty in the polar vortex response were completely eliminated, while the value for measure 2 roughly indicates how much spread would remain if all other sources of uncertainty, except that in the polar vortex response, were eliminated. Note that since these measures are based on standard deviations and therefore do not additively decompose the variance according to (4), measures 1 and 2 are not, in general, the same. We use these measures as they speak directly to the effects of stratospheric variability on confidence intervals derived from the CMIP5 multimodel ensemble, but we compare these measures with the variance-based measures of M2014 in Table 3.

\section{f. Significance testing}

Bootstrapping tests are used to assess the significance of, and uncertainties on, regression coefficients or 
TABLE 3. A comparison of measures of the influence of stratospheric polar vortex changes on different measures of tropospheric change: $\bar{u}$ at $700 \mathrm{hPa}$ and $60^{\circ}-65^{\circ} \mathrm{N}$ (green lines in Fig. 4e); 700-hPa $u$ averaged over the United Kingdom (green box in Fig. 5h); SLP averaged from $70^{\circ} \mathrm{N}$ to the pole (green circled region in Fig. 6h); and precipitation averaged over the United Kingdom and Spain (green boxes in Fig. 7h). Four different methods are used to quantify the reduction in CMIP5 model spread after removing the stratospheric contribution: $\mathrm{A}$ is the method used in the main text (i.e., subtracting the component from each model that is linearly related to $\bar{u}_{\mathrm{pv}}$ from an across-model regression); B is first performing sequential regressions to remove the component of model spread arising from spread in tropical upper-tropospheric warming and Arctic amplification (as in M2014) and then assessing the subsequent reduction in spread upon regressing out the contribution related to $\bar{u}_{\mathrm{pv}}$; and $\mathrm{C}$ and $\mathrm{D}$ are the same as $\mathrm{A}$ and $\mathrm{B}$, but using the polar vortex index of M2014 (the change in $\bar{u}$ at $10 \mathrm{hPa}$ and $70^{\circ}-80^{\circ} \mathrm{N}$ ). Note that for $\mathrm{B}$ and $\mathrm{D}$, unlike for $\mathrm{A}$ and $\mathrm{C}$, the component that is linearly related to the globally averaged changed in $T_{s}$ is not first regressed out from the fields to keep the methodology consistent with M2014. The first four columns quantify the reduction in $4 \sigma$ in percent and the second four columns quantify the reduction in $\sigma^{2}(\%)$. The final two columns quantify the difference between models on the extreme ends of the distribution (absolute value) relative to the $4 \sigma$ range, in \%, by the WEAK4x - STRONG4x difference and the CMIP5 regression times 10, respectively. Uncertainty ranges denoted by the subscript and superscript numbers are the 2.5th-97.5th percentiles calculated as described in section $3 \mathrm{f}$.

\begin{tabular}{|c|c|c|c|c|c|c|c|c|c|c|}
\hline \multirow[b]{2}{*}{ Index } & \multicolumn{4}{|c|}{$4 \sigma$} & \multicolumn{4}{|c|}{$\sigma^{2}$} & \multirow{2}{*}{$\frac{4 \sigma}{\text { CESML46 }}$} & \multirow{2}{*}{$\frac{4 \sigma}{\mathrm{CMIP} 5}$} \\
\hline & A & B & $\mathrm{C}$ & $\mathrm{D}$ & A & B & $\mathrm{C}$ & $\mathrm{D}$ & & \\
\hline $\bar{u}$ at $60^{\circ}-65^{\circ} \mathrm{N}$ & $10.1_{1.4}^{25.5}$ & $9.8_{0.6}^{28.6}$ & $13.2_{2.8}^{31.2}$ & $12.6_{1.5}^{35.7}$ & $19.2_{1.5}^{30.7}$ & $18.6_{0.6}^{31.3}$ & $24.6_{1.5}^{36.7}$ & $23.7_{1.5}^{35.7}$ & $23.1_{15.5}^{30.0}$ & $34.4_{13.1}^{56.0}$ \\
\hline$u$ at $700 \mathrm{hPa}$ (U.K. box) & $7.1_{0.4}^{25.0}$ & $6.5_{0.1}^{22.6}$ & $9.2_{0.5}^{12.9}$ & $8.5_{0.2}^{22.2}$ & $13.6_{0.6}^{50.3}$ & $12.6_{0.2}^{45.4}$ & $17.6_{0.6}^{49.7}$ & $16.1_{0.3}^{45.9}$ & $19.3_{10.5}^{27.7}$ & $28.8_{1.2}^{57.1}$ \\
\hline SLP (Arctic) & $8.5_{1.8}^{23.2}$ & $8.6_{1.3}^{21.5}$ & $10.9_{1.9}^{24.7}$ & $10.9_{2.0}^{25.5}$ & $16.3_{3.2}^{48.2}$ & $16.3_{2.2}^{44.3}$ & $19.9_{3.2}^{49.7}$ & $20.3_{3.3}^{51.2}$ & $30.6_{19.8}^{41.3}$ & $30.3_{2.8}^{58.3}$ \\
\hline Precipitation (U.K. box) & $3.1_{0.6}^{15.3}$ & $2.8_{0.3}^{14.4}$ & $3.4_{0.3}^{15.8}$ & $3.0_{0.4}^{14.8}$ & $6.1_{1.3}^{28.2}$ & $5.4_{0.6}^{25.2}$ & $6.6_{0.6}^{27.7}$ & $5.8_{0.7}^{25.4}$ & $11.8_{-1.6}^{26.0}$ & $17.8_{-12.6}^{43.7}$ \\
\hline Precipitation (Spain box) & $3.8_{0.7}^{15.1}$ & $3.5_{0.4}^{14.0}$ & $4.7_{0.7}^{14.9}$ & $4.4_{0.5}^{15.5}$ & $7.4_{1.2}^{29.3}$ & $6.9_{0.7}^{27.5}$ & $9.2_{1.0}^{28.4}$ & $8.5_{0.8}^{29.6}$ & $7.0_{-2.8}^{16.2}$ & $19.8_{-19.8}^{46.1}$ \\
\hline
\end{tabular}

differences. For the CMIP5 regression coefficient, this involves randomly resampling, with replacement, 35 models from the 35 available, and recomputing the regression coefficient $\beta_{X} 1000$ times. For the difference between two experiments, 1 and 2, with length $N_{\mathrm{y} 1}$ and $N_{\mathrm{y} 2}, N_{\mathrm{y} 1}$ years are resampled with replacement from experiment 1 , and $N_{\mathrm{y} 2}$ years are resampled with replacement from experiment 2 and the difference in their means is calculated. This is repeated 1000 times. For both the regression coefficients and the differences, the uncertainty is taken as the 2.5 th-97.5th percentile range and the quantity is considered significant if this range does not encompass zero (equivalent to significance at the $5 \%$ level for a two-sided test).

In Table 3 (see also Fig. 4e), confidence intervals are provided on the two measures of stratospheric influence on intermodel spread. In all cases, the reduction in spread is expressed as a percentage of the CMIP5 spread, but the uncertainties in the CMIP5 spread itself (i.e., the denominator) are not considered when providing this uncertainty estimate. For the second measure of stratospheric influence, that is, the difference between the WEAK4x and STRONG4x experiments (section $3 e$ ), the confidence interval is simply derived from the confidence interval on the difference between these two experiments as described above. For the first measure, that is, the reduction in spread obtained by regressing out the component related to $\bar{u}_{\mathrm{pv}}$, the uncertainty is derived by randomly sampling, with replacement, 35 models from the 35 available, and computing the reduction in spread obtained by regressing out the component related to $\bar{u}_{\mathrm{pv}}$ within this random sample. This is repeated 1000 times, and the 2.5 th-97.5th percentile range of the reduction in spread is used to obtain the confidence interval.

\section{Results}

We first show the overall anomalies in zonal-mean zonal wind for the NUDG4x - NUDG1x, STRONG4x NUDG1x, and WEAK4x - NUDG1x differences in Figs. 3a-c. These can be compared with the anomalies for the CMIP5 multimodel mean and the mean of the three most strengthening and three most weakening models in Figs. 3d-f. Aside from the fact that the $4 \times \mathrm{CO}_{2}$ experiments in CESML46 warm more than the CMIP5 RCP8.5 multimodel mean (because the $\mathrm{CO}_{2}$ perturbation is bigger), resulting in a greater strengthening of the subtropical jet, these experiments are successful in mimicking the range of high-latitude stratospheric anomalies that are seen in CMIP5. We now proceed to examine the influence of these stratospheric perturbations on the troposphere below and compare with the CMIP5 across-model regressions for various fields.

\section{a. Zonal-mean zonal wind}

The influence that this range in polar vortex responses may have on the zonal-mean zonal wind in the troposphere can be assessed from Fig. 4. Note that here, and in all subsequent analyses, we present results from the perspective of a weakening of the polar vortex. The regression, across models, of the future - past difference in $\bar{u}$ 
(a) NUDG4x-NUDG1x

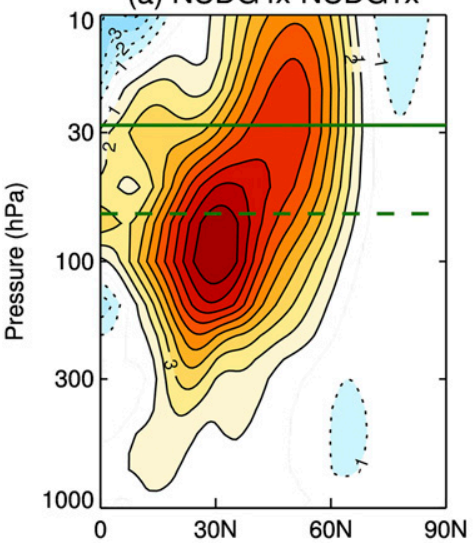

(b) STRONG4x-NUDG1x

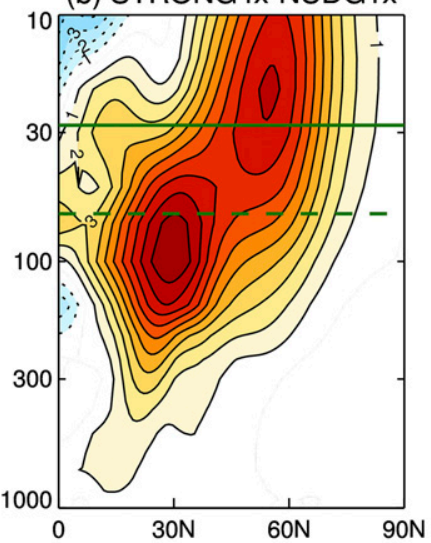

(c) WEAK4x-NUDG1x

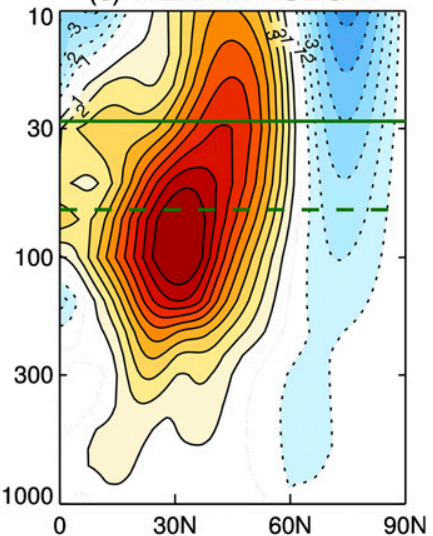

(d) CMIP5 MMM

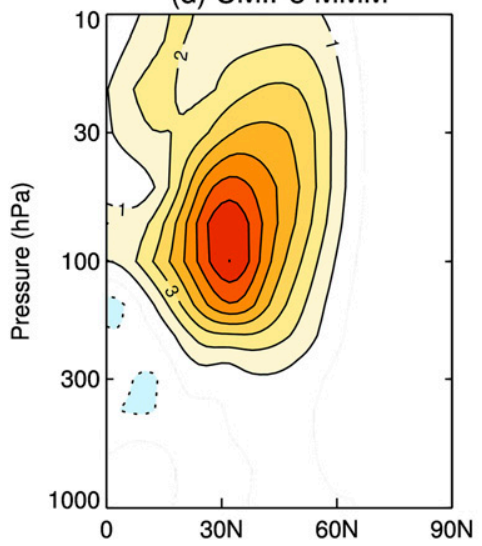

(e) CMIP5 strongest

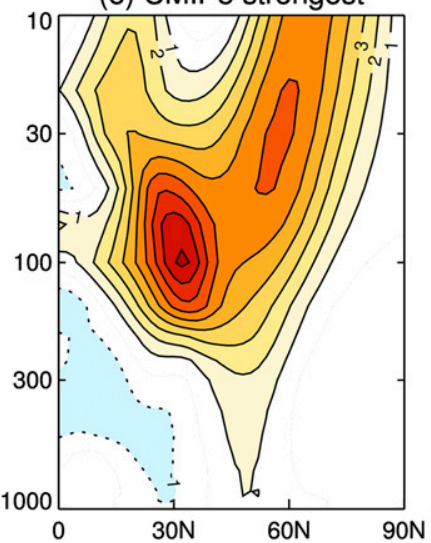

(f) CMIP5 weakest

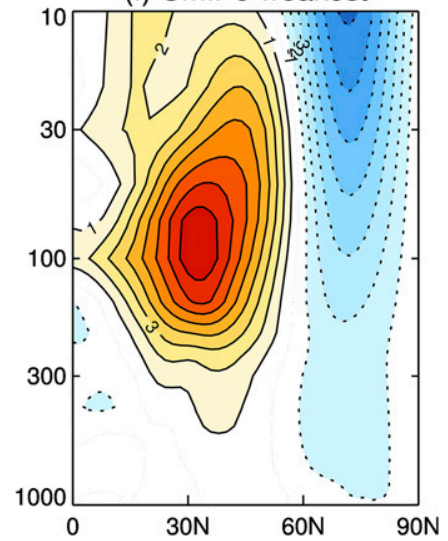

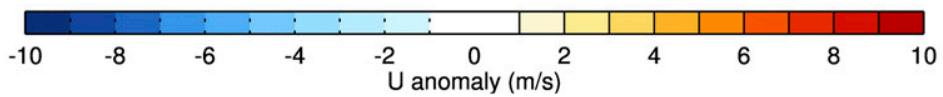

FIG. 3. DJF-averaged zonal-mean zonal wind anomalies for CESML46 (a) NUDG4x - NUDG1x, (b) STRONG4x - NUDG1x, (c) WEAK4x - NUDG1x, (d) the CMIP5 multimodel-mean future - past difference, (e) the future - past difference for the mean of the three models with the greatest increase in $\bar{u}_{\mathrm{pv}}$ (MIROC5, GFDL CM3, and MIROC-ESM), and (f) the future - past difference for the mean of the three models with the greatest decrease in $\bar{u}_{\mathrm{pv}}$ (MRI-CGCM3, MPI-ESM-MR, and MPI-ESM-LR). The solid and dashed green lines in (a)-(c) denote the tapering region for the stratospheric nudging.

onto $\bar{u}_{\mathrm{pv}}$; that is, $\beta_{\bar{u}}$ multiplied by $-\Delta \bar{u}_{\mathrm{pv}}$, with $\Delta \bar{u}_{\mathrm{pv}}=10 \mathrm{~m} \mathrm{~s}^{-1}$ [see (4)] is shown in Fig. 4a (note the nonlinear contour interval compared to Fig. A1g). This shows very similar results to M2014 (see their Fig. 4e) and indicates that with a greater weakening of the stratospheric vortex comes easterly anomalies in the high-latitude troposphere with westerly anomalies to the south. Figure $4 \mathrm{a}$ indicates that, with all else being equal, we should expect the models on the extreme weakening end of the CMIP5 scale to differ from those on the extreme strengthening end by an easterly anomaly of around $-0.5 \mathrm{~m} \mathrm{~s}^{-1}$ on the poleward side of the jet and a westerly anomaly of around $0.5 \mathrm{~m} \mathrm{~s}^{-1}$ on the equatorward side of the jet.
This can be compared with the difference between WEAK4 $\mathrm{x}$ and STRONG4x where extreme weakening and strengthening anomalies of the polar vortex have been artificially imposed (Fig. 4b). By construction, Figs. $4 \mathrm{a}$ and $4 \mathrm{~b}$ look very similar above the nudging level (green horizontal lines in Fig. 4b). In addition to this, in agreement with the hypothesis that the tropospheric anomalies are produced as a response to the stratospheric anomalies, the imposition of these polar vortex anomalies within CESML46 produces quantitatively similar anomalies in the troposphere as well. In WEAK4x, relative to STRONG4x, there is an easterly anomaly of about $-0.5 \mathrm{~m} \mathrm{~s}^{-1}$ extending to the surface on 
the poleward side of the jet and a westerly anomaly farther equatorward. Although the anomalies in WEAK4x - STRONG4x are slightly weaker than those from the CMIP5 regression and the easterly anomalies do not extend to as high latitudes, the WEAK4x STRONG4 $\mathrm{x}$ difference lies well within the uncertainty range of the CMIP5 regression (Fig. 4c). Given that, within one model, quantitatively similar results to that inferred from the regression across CMIP5 models can be obtained by imposing anomalies in the stratospheric polar vortex, it can be concluded that the downward influence inferred from such CMIP5 regressions likely does represent a true downward influence of the stratosphere on the troposphere below.

The green lines in Fig. 4d show the FREE4x FREE1 $x$ and NUDG4x - NUDG1x differences. The only region where nudging has a significant influence on the $4 \times \mathrm{CO}_{2}$ minus $1 \times \mathrm{CO}_{2}$ difference is poleward of $80^{\circ} \mathrm{N}$. Elsewhere, and in all subsequent analyses, an influence of nudging on the response is not apparent. In any case, we will always be comparing one nudged run with another so any discrepancies related to the presence of nudging should be largely cancelled out. ${ }^{2}$

Figures $4 \mathrm{~d}$ and $4 \mathrm{e}$ also allow us to assess the magnitude of the stratospheric influence relative to the overall CMIP5 spread (see section $3 e$ ). The $4 \sigma$ range of the CMIP5 models is around $2 \mathrm{~m} \mathrm{~s}^{-1}$ (thin solid lines in Fig. 4d). After removing the component of the $\bar{u}$ anomalies that is linearly related to $\bar{u}_{\mathrm{pv}}$, this $4 \sigma$ range reduces slightly (dotted lines in Fig. 4d). As a fraction of the original CMIP5 $4 \sigma$ range, this reduction is on the order of $10 \%$, maximizing around $65^{\circ} \mathrm{N}$ and just equatorward of $40^{\circ} \mathrm{N}$ (Fig. 4e). In our idealized setup, the difference between the anomalies in WEAK4x and STRONG4x is a clearly visible portion of the CMIP5 spread (Fig. 4d) with the magnitude of the anomalies equivalent to about $20 \%$ of the CMIP5 spread on the poleward side of the jet and about $10 \%$ of the CMIP5 spread on the equatorward side of the jet. What Fig. 4e tells us is that, for example, just poleward of $65^{\circ} \mathrm{N}$, we expect the difference between the polar vortex anomalies on the extreme weakening and extreme strengthening end of the CMIP5 range to give rise to a difference that is on the order of $20 \%$ of the CMIP5 $4 \sigma$ range. However, among the CMIP5 models that do not all

\footnotetext{
${ }^{2}$ Comparison of the difference between the responses in WEAK4x and STRONG4x (blue and red in Fig. 4d) and that in NUDG4x may suggest some nonlinearity; that is, poleward of around $60^{\circ} \mathrm{N}$, the response in STRONG4x is more different from the response in NUDG4 $x$ than the response in WEAK $4 x$ is. However, this is not robust enough to consider the two halves of the STRONG4 $\mathrm{x}$ and WEAK4 $\mathrm{x}$ experiments separately.
}

exhibit such extreme vortex anomalies, and in the presence of all other factors that may give rise to intermodel spread, the removal of the polar vortex contribution from each model only amounts to a reduction in the CMIP5 $4 \sigma$ range of around $10 \%$.

\section{b. Latitude-longitude 700-hPa zonal wind}

For this and subsequent latitude-longitude fields we use a uniform nine-panel format in the figures. Considering 700-hPa zonal wind u700 (Fig. 5), CESML46 under $4 \times \mathrm{CO}_{2}$ (Fig. 5b) exhibits a generally similar response to the CMIP5 multimodel mean (Fig. 5a), albeit with a greater magnitude as a result of greater warming. For example, the enhanced westerlies at the extension of the Atlantic jet over Europe and the easterlies over North Africa (Woollings and Blackburn 2012; Simpson et al. 2014; Zappa et al. 2015) are present in both, along with enhanced westerlies west of California (Neelin et al. 2013; Seager et al. 2014b) and east of Japan. The CESML46 response to $4 \times \mathrm{CO}_{2}$ is extremely similar in the nudged and free configurations (cf. Figs. 5b and 5c). Next, the CMIP5 regression, $\beta_{\mathrm{u} 700} \times-\Delta \bar{u}_{\mathrm{pv}}$, with $\Delta \bar{u}_{\mathrm{pv}}=10 \mathrm{~m} \mathrm{~s}^{-1}$, is shown in Fig. $5 \mathrm{~d}$ and demonstrates that the easterly anomaly seen in the high latitudes in the zonal mean (Fig. 4a) is somewhat concentrated over the Atlantic sector, akin to the tropospheric response to intraseasonal stratospheric variability (Baldwin and Dunkerton 2001; Hitchcock and Simpson 2014). This CMIP5 regression can be compared with the WEAK4x STRONG4x difference in Fig. 5e and the significance of any differences between them can be inferred from Fig. 5f. In general, the pattern of stratospheric influence obtained from WEAK4x - STRONG4x agrees with that inferred from the CMIP5 regression (one possible exception is over western Canada and the southern United States).

Figures $5 \mathrm{~g}-\mathrm{i}$ provide indications of the magnitude of the stratospheric influence relative to the overall CMIP5 spread. Over Scotland and the North Sea, the CMIP5 $4 \sigma$ range (Fig. $5 \mathrm{~g}$ ) is around $5 \mathrm{~m} \mathrm{~s}^{-1}$, which can be compared with the anomalies in Fig. 5d and 5e of around $1-1.5 \mathrm{~m} \mathrm{~s}^{-1}$. After removing the component of the u 700 anomalies in CMIP5 that are related to $\bar{u}_{\mathrm{pv}}$ by linear regression, the CMIP5 $4 \sigma$ range drops by, at most, around $8 \%$ (Fig. $5 \mathrm{~h}$ ). However, the magnitude of the WEAK $4 x$ - STRONG4 $x$ difference amounts to about $20 \%$ of the CMIP5 $4 \sigma$ range over the eastern portion of the North Atlantic and northern Europe, and even more farther to the east where the $4 \sigma$ range of the CMIP5 models is lower.

Overall, the agreement between the WEAK4x STRONG4x difference and the CMIP5 regression indicates that the u700 anomalies found in the CMIP5 
(a) CMIP5 $-\beta_{u} \times 10$

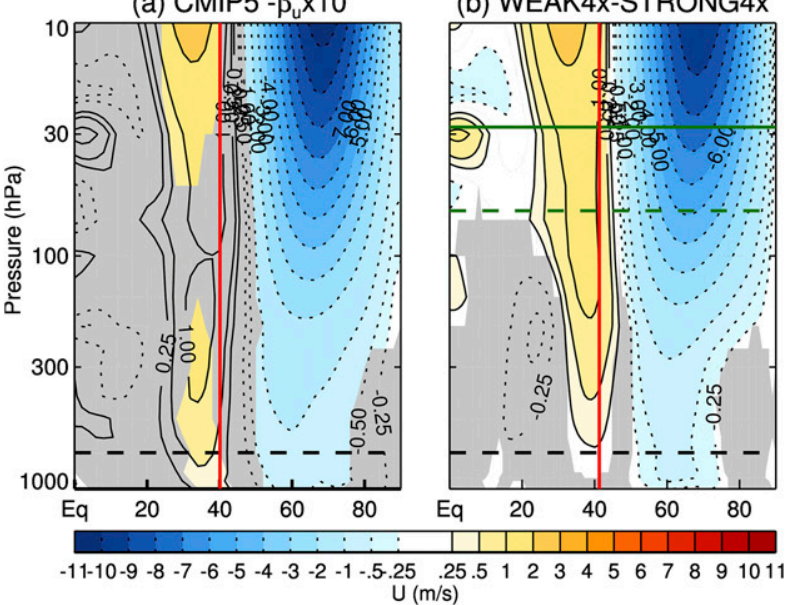

(c) $700 \mathrm{hPa} U,-\beta_{4} \times 10 /$ WEAK4x-STRONG4x

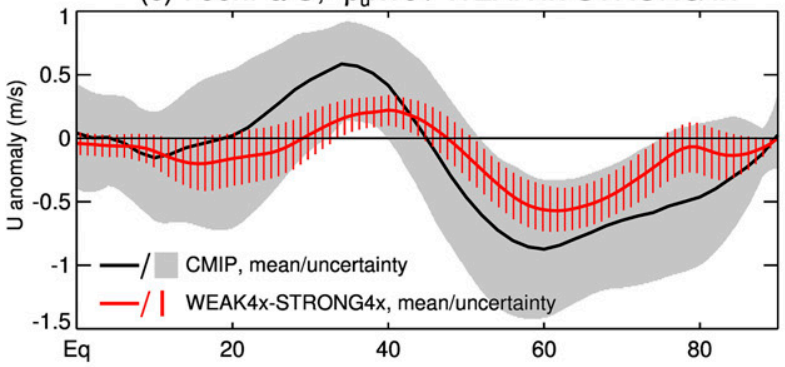

(d) $700 \mathrm{hPa} U$, Future-Past or $4 \mathrm{xCO}_{2}-1 \times \mathrm{xO}_{2}$

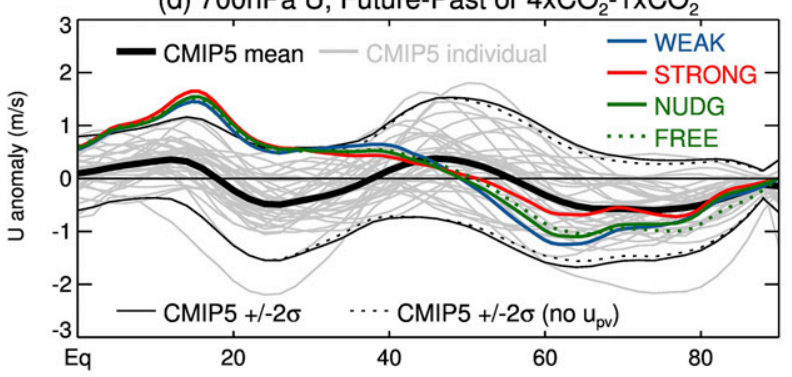

(e) $\%$ of $4 \sigma$

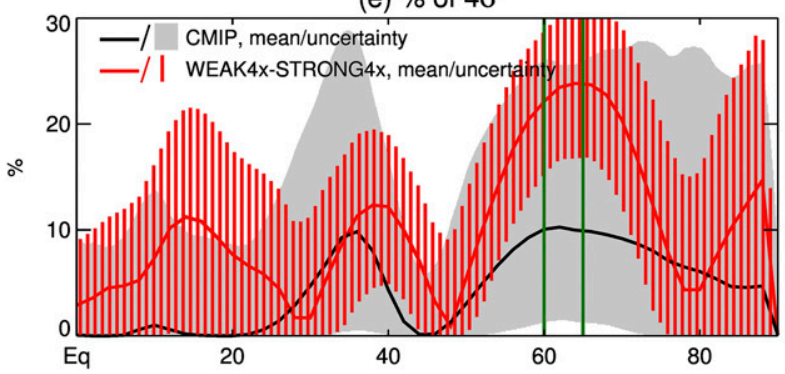

FIG. 4. DJF-averaged zonal-mean zonal wind. (a) The regression of the future - past difference onto $\bar{u}_{\mathrm{pv}}$ for the 35 CMIP5 models $\left(-\beta_{u} \times 10\right)$. (b) The difference between WEAK4x and STRONG4x. Gray shaded regions in (a) and (b) are not significant at the 5\% level by the methods outlined in section $3 \mathrm{f}$. (c) The $700-\mathrm{hPa}$ values of $-\beta_{u} \times 10$ and the WEAK4x - STRONG4x difference along with their uncertainties. (d) The future - past difference for the individual CMIP5 models (gray) and the multimodel mean (thick black) along regression, at least in the North Atlantic and over northern Europe, are likely produced as a response to the different zonal-mean climatological changes in the stratosphere. Removal of the stratospheric influence by linear regression removes around $5 \%-10 \%$ of the CMIP5 model spread in the North Atlantic but, all else being equal, we should expect the difference between the polar vortex states on the extreme weakening and strengthening ends of the CMIP5 range to result in zonal wind anomalies over the North Atlantic and Europe that are on the order of $20 \%$ of the CMIP5 spread.

\section{c. Sea level pressure}

The SLP response can be examined through Fig. 6 . The main features of the CMIP5 predicted response in SLP (Fig. 6a) are reproduced in the CESML46 response to $4 \times \mathrm{CO}_{2}$, both in the free-running (Fig. 6b) and nudged (Fig. 6c) configurations, albeit with a greater magnitude. The CMIP5 regression, $\beta_{\mathrm{SLP}} \times-\Delta \bar{u}_{\mathrm{pv}}$, (Fig. 6d) shows that a relative weakening of the stratospheric polar vortex is accompanied by a relative increase (reduced decrease) in SLP over the Arctic. It is also accompanied by a relative decrease in SLP at lower latitudes, but this is, for the most part, not statistically significant. In the WEAK4x - STRONG4x difference (Fig. 6e), very similar features are seen to those in the CMIP5 regression with an increased SLP over the polar cap and a reduced SLP farther south, with greater significance in the southern anomalies compared to the CMIP5 regression. The WEAK4x - STRONG4x polar cap anomalies are slightly shifted off the pole relative to those from the CMIP5 regression, leading to some anomalies over Eurasia and North America in WEAK4 $\mathrm{x}$ - STRONG4 $\mathrm{x}$ that lie outside of the CMIP5 uncertainty range (Fig. 6f). Over the polar cap,

with the $\pm 2 \sigma$ range before (solid) and after (dotted) regressing out the component of the future - past difference in $\bar{u}$ that is linearly related to $\bar{u}_{\mathrm{pv}}$. Red and blue lines show the STRONG4x NUDG1 $\mathrm{x}$ and WEAK4x - NUDG1x differences, respectively, while green dotted and solid lines show the FREE4x - FREE1x and NUDG4x - NUDG1x differences, respectively. (e) Black shows the reduction in the $4 \sigma$ range of the CMIP5 future - past difference after regressing out the component related to $\bar{u}_{\mathrm{pv}}$, and red shows the difference between WEAK4x and STRONG4x, both expressed as a percentage of the CMIP5 $4 \sigma$ range. Uncertainty ranges are calculated as outlined in section $3 \mathrm{f}$. Black dashed line in (a) and (b) shows the 700-hPa level used in (c)-(e), and red lines show the past (or $1 \times \mathrm{CO}_{2}$ ) climatological jet latitude at $700 \mathrm{hPa}$. Solid and dashed green lines in (b) denote the tapering region for the stratospheric nudging, and green lines in (e) show the averaging region used for Table 3 . 
(a) CMIP5, Future-Past

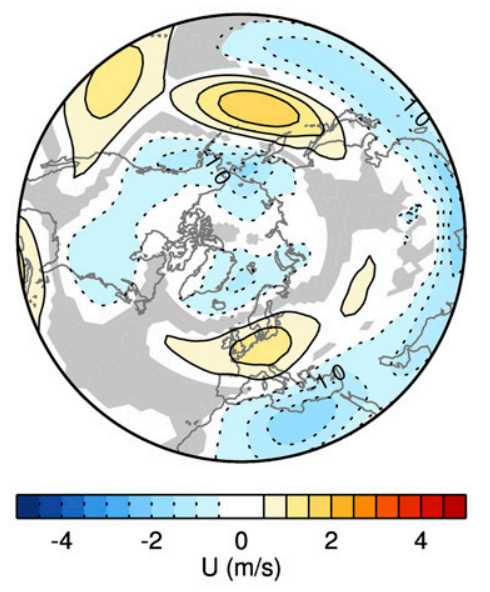

(d) CMIP5 $-\beta_{\mathrm{u} 700} \times 10$

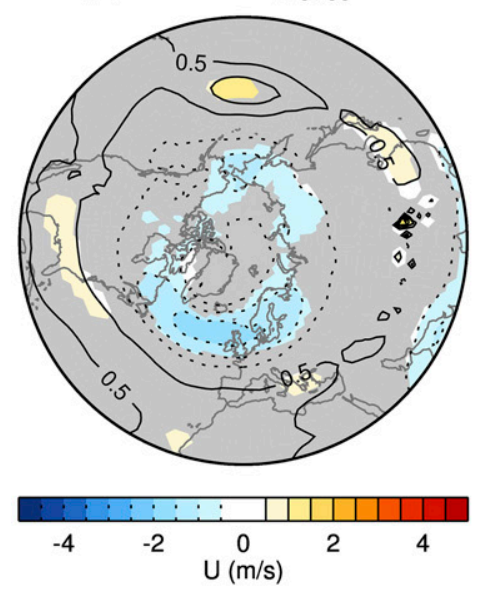

(g) CMIP5 4xo

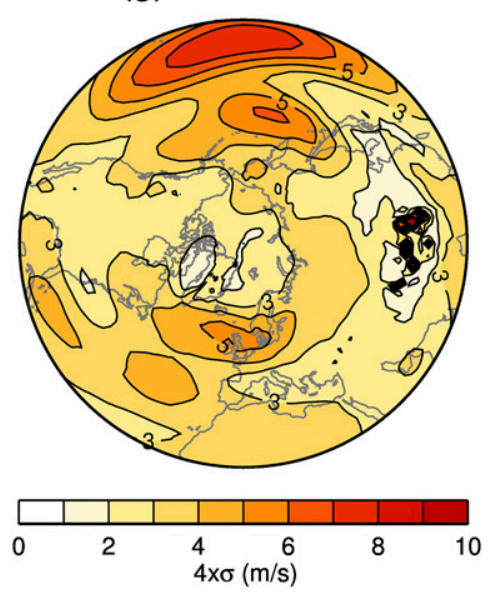

(b) FREE4x-FREE1x

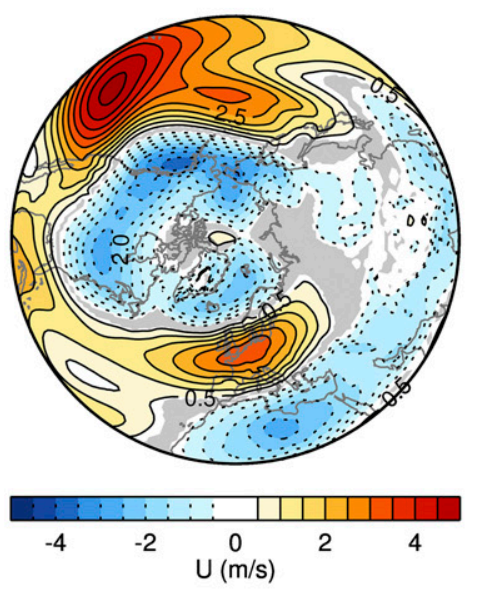

(e) WEAK4x-STRONG4 $x$

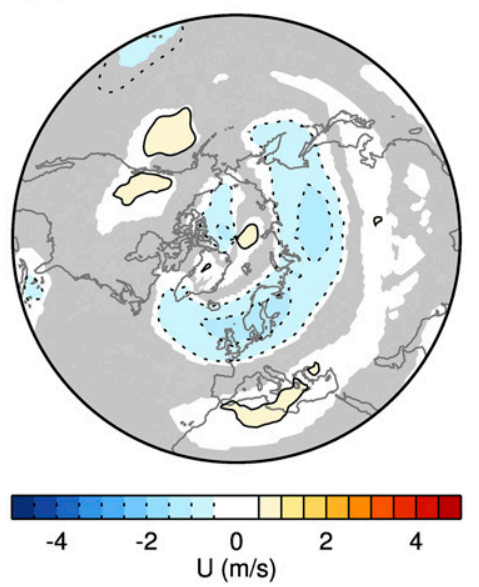

(h) $\%$ of $4 x \sigma$, CMIP5

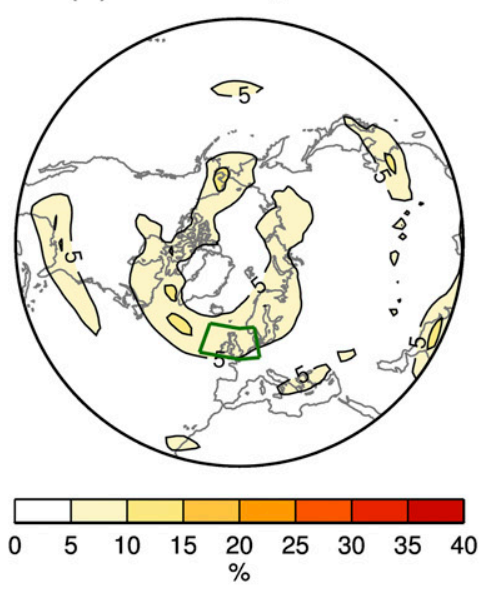

(c) NUDG4x-NUDG1x

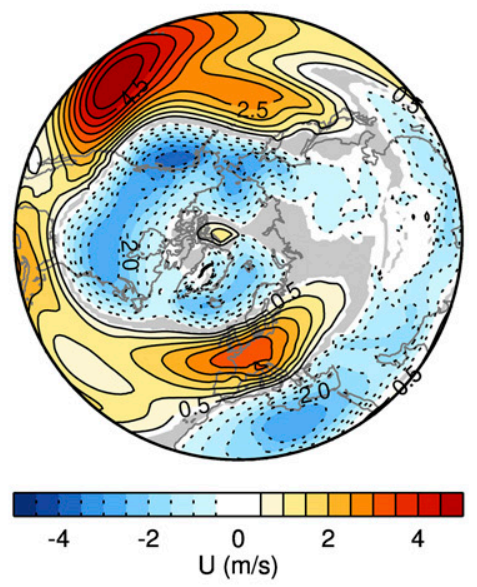

(f) Difference (e)-(d)

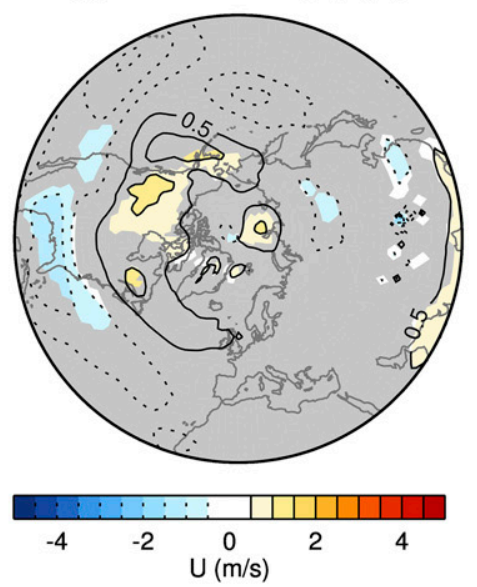

(i) $\%$ of $4 x \sigma$, WEAK $4 x-S T R O N G 4 x$

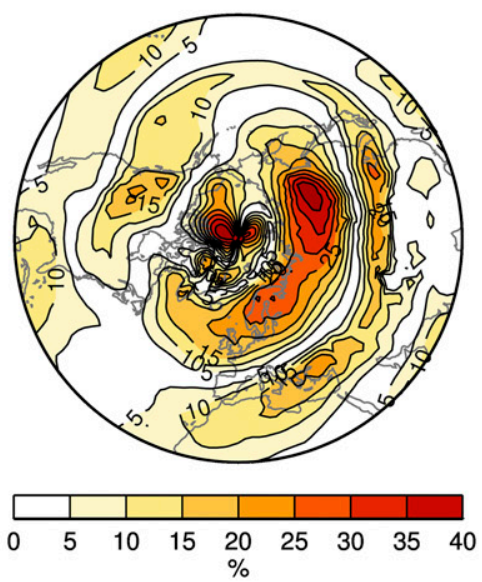

FIG. 5. DJF 700-hPa zonal wind. (a) CMIP5 multimodel-mean future - past difference, (b) FREE4x - FREE1x, and (c) NUDG4x NUDG1x differences. (d) The CMIP5 regression onto $\bar{u}_{\mathrm{pv}}\left(-\beta_{\mathrm{u} 700} \times \Delta \bar{u}_{\mathrm{pv}}\right)$, (e) the WEAK4x - STRONG4x difference, and (f) the difference between (e) and (d). (g) The CMIP5 $4 \sigma$ range, (h) the reduction in the CMIP5 $4 \sigma$ range after regressing out the component related to $\bar{u}_{\mathrm{pv}}$, and (i) the WEAK4x - STRONG4x difference, both expressed as a percentage of the CMIP5 4 $\sigma$ range. In (a)-(e), gray shaded regions are not significant at the $5 \%$ level by the measures outlined in section $3 \mathrm{f}$, and in (f), gray shaded regions denote where the WEAK $4 \mathrm{x}-$ STRONG4 $\mathrm{x}$ difference lies within the uncertainty range of $-\beta_{\mathrm{u} 700} \times 10$. Colored and white regions are significant. The region encompassed by green lines in (h) is used in Table 3. 
(a) CMIP5, Future-Past

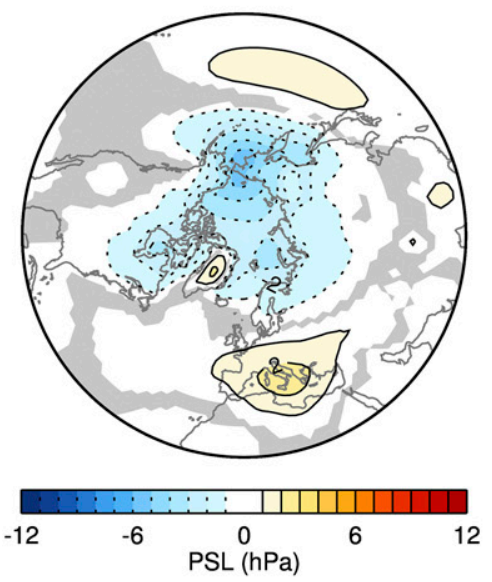

(d) CMIP5 - $\beta_{\mathrm{PSL}} \times 10$

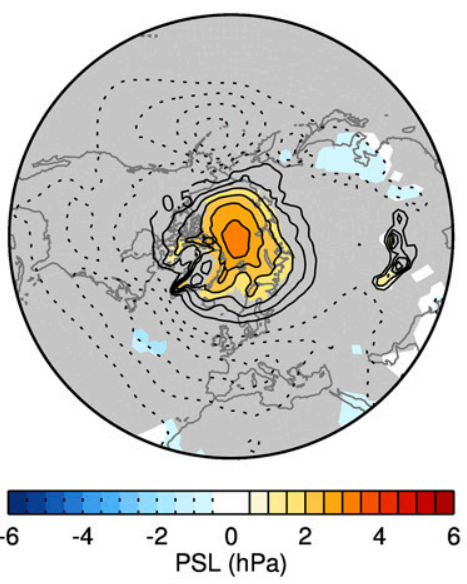

(g) CMIP5 4xo

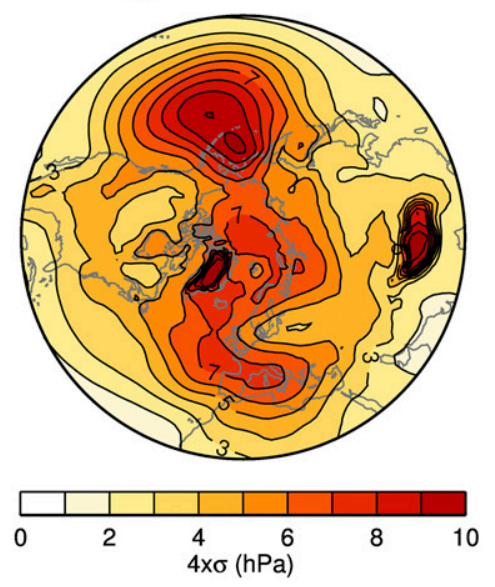

(b) FREE4x-FREE1 $x$

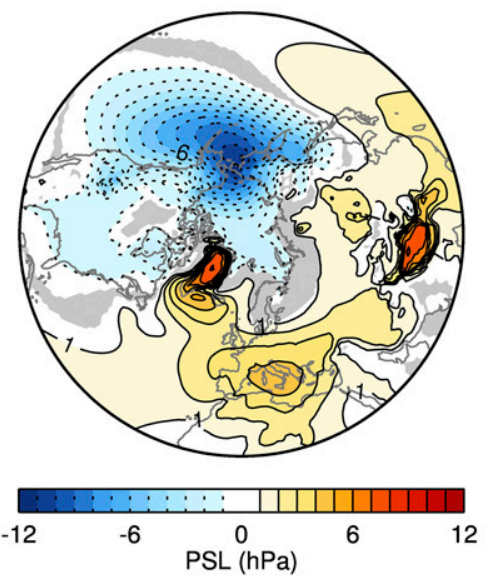

(e) WEAK4x-STRONG4x

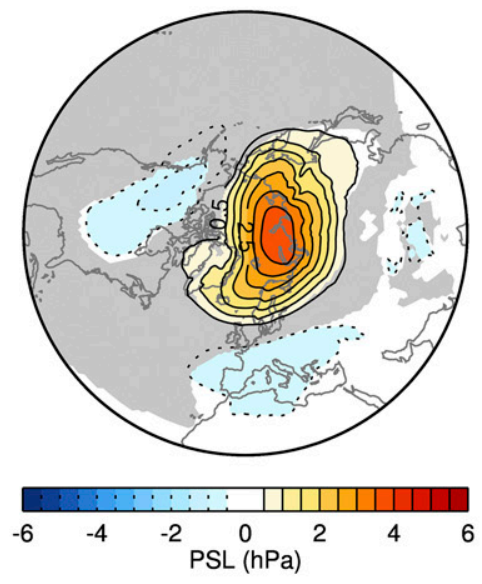

(h) $\%$ of $4 \times \sigma$, CMIP5

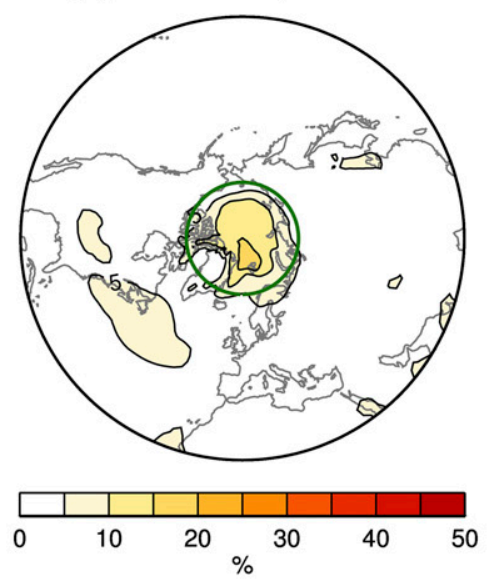

(c) NUDG4x-NUDG1x

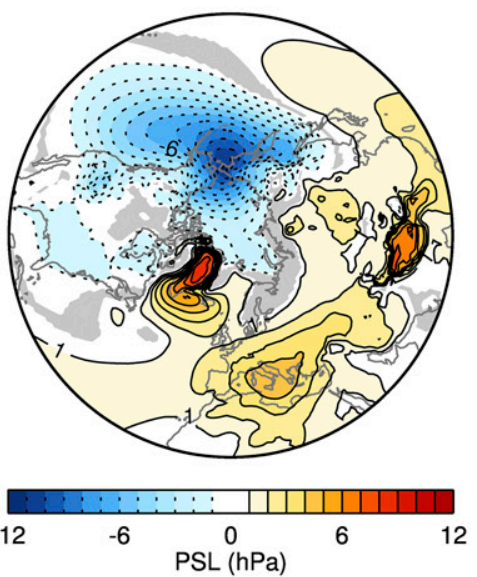

(f) Difference (e)-(d)

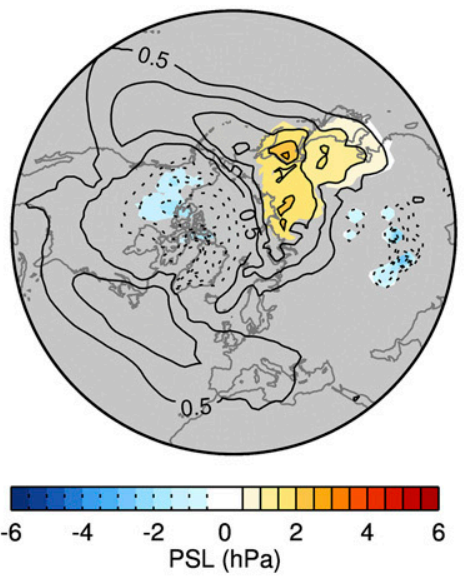

(i) $\%$ of $4 x \sigma$, WEAK $4 x-S T R O N G 4 x$

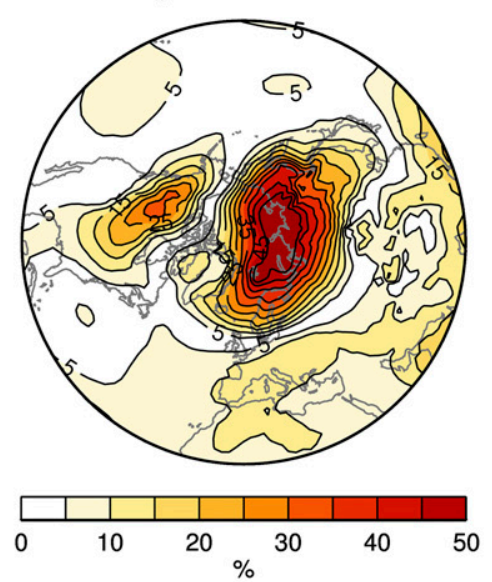

FIG. 6. As in Fig. 5, but for sea level pressure.

regressing out the SLP anomalies associated with $\bar{u}_{\mathrm{pv}}$ reduces the CMIP5 $4 \sigma$ range by up to $15 \%$. The WEAK4 $\mathrm{x}$ - STRONG4 $\mathrm{x}$ difference (and the CMIP5 regression coefficient) suggests that, all else being equal, the difference between the polar vortex changes at the extreme weakening and extreme strengthening ends of the CMIP5 range gives rise to polar cap SLP anomalies that are on the order of $50 \%$ of the CMIP5 spread. 
(a) CMIP5, Future-Past

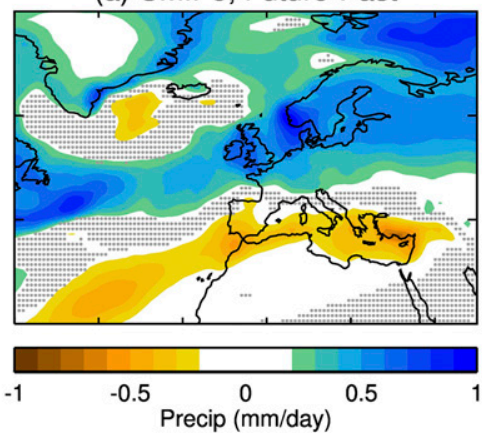

(d) CMIP5 - $\beta_{\mathrm{pr}} \times 10$

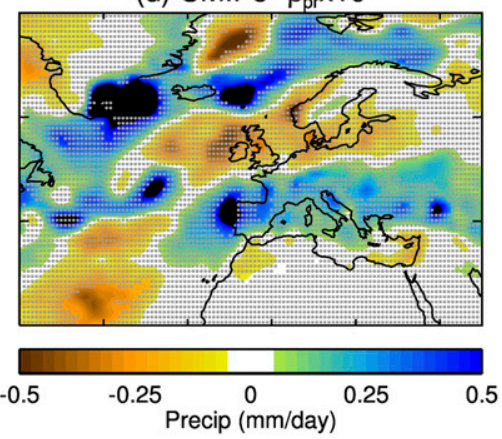

(g) CMIP5 4xo

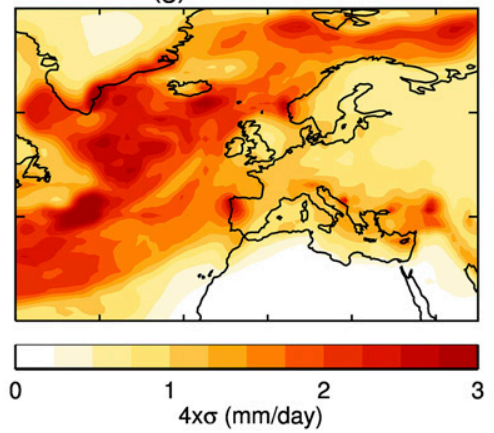

(b) FREE4x-FREE1x

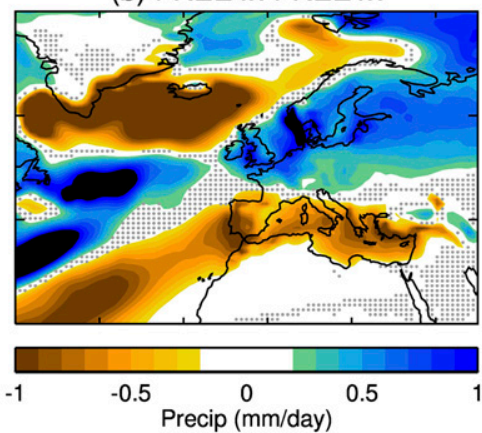

(e) WEAK4x-STRONG4x

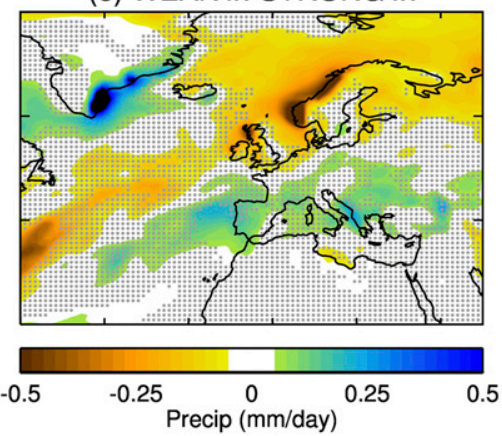

(h) $\%$ of $4 \sigma$, CMIP5
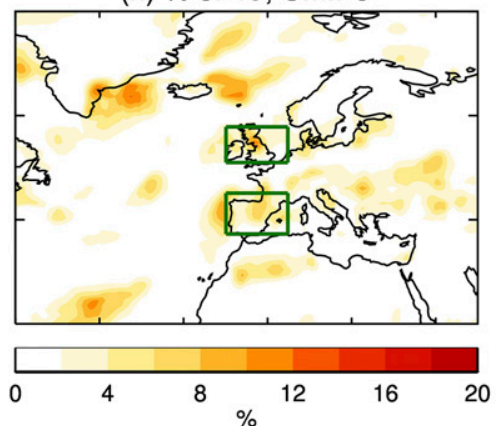

(c) NUDG4x-NUDG1x

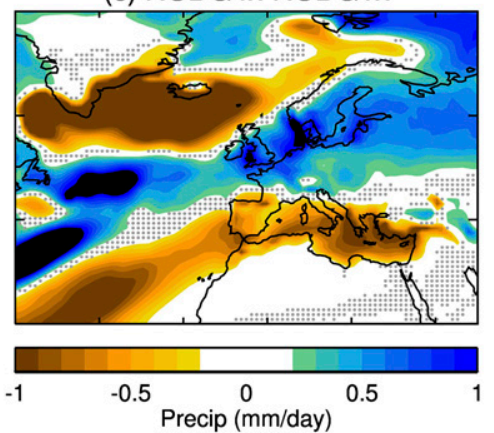

(f) Difference (e)-(d)

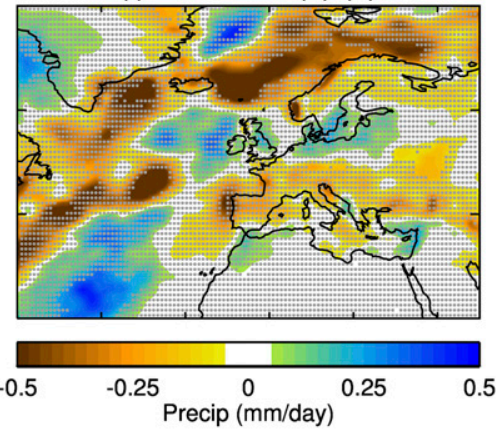

(i) $\%$ of $4 x \sigma$, WEAK $4 x-S T R O N G 4 x$

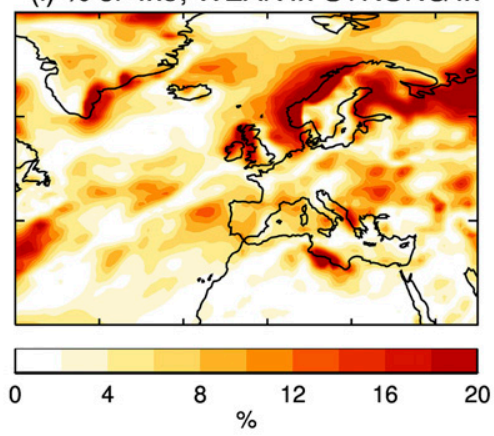

FIG. 7. As in Fig. 5, but for precipitation. Note the different method of signifying significance that allows the patterns of precipitation change to be seen more clearly given the small patchy regions of significance. Stippled regions in (a)-(f) are not significant at the $5 \%$ level.

\section{d. Precipitation}

Scaife et al. (2012) argued for an important role for stratospheric circulation on future European precipitation (pr) change in their high-top-low-top comparisons. This can be examined through Fig. 7. The CMIP5 multimodel mean shows a drying over the Mediterranean Sea and southern Mediterranean land regions along with a wetting over northern Europe (Fig. 7a). Similar features but with enhanced amplitude are seen in the free (Fig. 7b) and nudged (Fig. 7c) $4 \times \mathrm{CO}_{2}$ experiments. The CMIP5 regression, $\beta_{\mathrm{pr}} \times-\Delta \bar{u}_{\mathrm{pv}}$, suggests that a weakening of the polar vortex is accompanied by relative drying over northern Europe and relative wetting to the south over Spain, southern France, Italy, and Greece, although note the limited areas of significance (Fig. 7d). The WEAK4x - STRONG4x experiments corroborate the relative drying (wetting) pattern north (south) over Europe that accompanies a relative weakening of the polar vortex. Given the large uncertainty on the CMIP5 regression coefficient, the WEAK4x STRONG4x difference, for the most part, also agrees with the CMIP5 regression within the uncertainty limits. One place where they do differ is east of Iceland, where the regression coefficient suggests a weakening of the vortex should be accompanied by an increased precipitation whereas the WEAK4x - STRONG4x difference suggests it should be accompanied by a decrease. 
Nevertheless, over European land regions, they agree. They also agree that a relative weakening of the polar vortex will result in a rather substantial enhanced wetting on the southeastern coast of Greenland, which may have implications for future changes in Greenland mass balance (Box et al. 2013).

The CMIP5 $4 \sigma$ range (Fig. $7 \mathrm{~g}$ ) shows relatively greater intermodel spread in southern Europe around the zero line of the multimodel-mean future - past difference (Fig. 7a) as well as over the western portion of Scandinavia. In southern Europe, after regressing out the component related to $\bar{u}_{\mathrm{pv}}$ in CMIP5, the $4 \sigma$ range is reduced by around $8 \%$. However, the WEAK $4 \mathrm{x}-$ STRONG4x difference suggests that, all else being equal, the difference in the polar vortex between the models at the extreme weakening and extreme strengthening of the CMIP5 range could give rise to precipitation differences in southern Europe, the United Kingdom, and Scandinavia that are on the order of $20 \%$ of the CMIP5 model spread.

\section{e. Sensitivity to methodology}

Throughout this analysis we have made subjective choices. In particular, our choice of polar vortex index $\left(60^{\circ}-75^{\circ} \mathrm{N}\right.$ and $\left.10-\mathrm{hPa} \bar{u}\right)$ was based on the region of largest model spread (Fig. 1d) and differs from that of M2014, who used $70^{\circ}-80^{\circ} \mathrm{N}$ and $10-\mathrm{hPa} \bar{u}$. The impact of this choice on the conclusions regarding the extent to which the spread is reduced upon regressing out the stratospheric contribution can be assessed by comparing columns $\mathrm{A}$ and $\mathrm{C}$ in Table 3. The impact is minimal but the stratospheric influence is slightly greater when the M2014 index is used. It is also possible that there are other sources of stratospheric uncertainty not captured by either of these indices, such as model differences in the extent to which anomalies in the polar vortex reach down to the lower stratosphere, where they are likely to have the most impact, or indeed, intermodel differences in the coupling strengths between the stratosphere and troposphere. Assessing this potential additional contribution to tropospheric uncertainty is beyond the scope of this study, but additional investigation into this is necessary to fully understand all the stratospheric sources of uncertainty.

Our measure of model spread was $4 \sigma$. M2014 instead used variance $\sigma^{2}$ and also first regressed out the component of intermodel spread associated with tropical upper-tropospheric warming and Arctic amplification before assessing the subsequent reduction in $\sigma^{2}$. These are, again, subjective choices and the impact on the conclusions can be assessed from Table 3. The prior regressions onto tropical upper-tropospheric warming and Arctic amplification have very little impact on the reduction in spread achieved by regressing out the stratospheric contribution (cf. columns A and B in Table 3 ). The choice of measure of model spread has a bigger impact, with the reduction in $\sigma^{2}$ being somewhere between 1.5 and 2 times the reduction in $4 \sigma$ (cf. the first four columns with the second four columns in Table 3 ). This difference is to be expected from these different measures and does not reflect any particular properties of the response.

Finally, while we have used our CESML46 experiments to provide a measure of the difference between models on opposite ends of the CMIP5 range, an equivalent measure could be obtained from the CMIP5 regression directly by using the CMIP5 regression coefficient multiplied by $\Delta \bar{u}_{\mathrm{pv}}=10 \mathrm{~m} \mathrm{~s}^{-1}$. This can be compared with the CESML46 measure for particular regions in the last two columns of Table 3 [or could be assessed from the ratios of panels (d) to (g) in Figs. 5, 6, and 7].

\section{Discussion and conclusions}

Prior evidence, both from observational and model assessments of stratospheric influence on the troposphere and from comparison of the climate change response between different models, has indicated that the simulation of stratospheric change represents a potential source of uncertainty in projections of tropospheric climate change.

A number of previous studies have inferred, either from comparison of different model versions (Shindell et al. 1999; Sigmond et al. 2008; Scaife et al. 2012; Karpechko and Manzini 2012) or from multimodel intercomparisons (M2014), that the way in which the stratospheric polar vortex responds in the future is connected to aspects of $\mathrm{NH}$ winter tropospheric circulation change. If this connection were to represent a downward influence of the stratosphere on the troposphere below then, given the wide spread among models in their predictions of stratospheric vortex change shown here and elsewhere (M2014; Butchart et al. 2010), the simulation of stratospheric change may represent a potential source of uncertainty on tropospheric climate change.

Our aim has been to complement these existing studies by performing idealized experiments, within one model, where stratospheric zonal-mean vortex states that mimic the CMIP5 range have been artificially imposed alongside an increase in GHGs, using a nudging methodology. The advantage of this is that it is only the stratospheric zonal-mean vortex anomalies that differ between model experiments, by construction. This allows for a clean demonstration and quantification of the influence of differences in the stratospheric zonalmean climatology on the troposphere below and an 
unambiguous demonstration of the presence of a causal link between stratospheric vortex change and the tropospheric circulation. These experiments should capture the influence of the climatological zonal-mean stratospheric boundary conditions on the troposphere below, but, given their design, there will necessarily be aspects of the stratospheric influence that they cannot capture. In particular, the nudging of the zonal-mean stratospheric winds and temperatures toward a seasonally varying climatological state means that transient large-amplitude events such as SSWs or reflection of planetary waves from anomalous vortex configurations (Perlwitz and Harnik 2003; Shaw et al. 2010) cannot be captured. This means that the influence of these aspects on the wintertime climatology will not be fully represented and it also precludes an investigation of subseasonal variability in these simulations. It should also be noted that we performed our investigation under a $4 \times \mathrm{CO}_{2}$ climate which is warmer than the end of the century under RCP8.5 in the CMIP5 models that we compare against. This would not be a fair comparison if the influence of the stratospheric perturbations changed as warming progresses, but the similarity between the stratospheric influence in our experiments and that inferred from CMIP5 suggests this is not so.

In terms of the stratospheric influence on wintertime climatological circulation change, good agreement is found between these experiments and the stratospheric influence inferred from linear regression across different models (M2014). This confirms that (i) the stratospheric influence inferred from such linear regressions is indeed a true downward influence of the stratospheric vortex change on the troposphere below and (ii) a substantial component of the stratospheric contribution to intermodel spread in the wintertime climatological change in the troposphere arises from the change in the zonal-mean climatological stratospheric boundary conditions.

To summarize the main features of the stratospheric influence found in these experiments, a relative weakening of the stratospheric polar vortex is accompanied by an easterly anomaly in zonal-mean zonal wind $\bar{u}$ in the NH mid-to-high latitudes and a westerly anomaly farther south (i.e., a relative equatorward shifting of the tropospheric midlatitude westerlies). This stratospheric influence on the tropospheric westerlies is fairly localized in the North Atlantic sector and over Europe, as is often found with stratospheric influences on the troposphere (Baldwin and Dunkerton 2001; Hitchcock and Simpson 2014). It is accompanied by a relative increase in Arctic SLP (reduced decrease) along with a relative decrease (reduced increase) farther to the south, a pattern similar to that of the negative phase of the North Atlantic Oscillation and/or northern annular mode. The dominant effect of this stratospheric influence on regional climate is found over Europe and the Mediterranean region where, accompanying the equatorward shifting of the Atlantic-European westerlies in association with a weakened vortex, precipitation is found to exhibit a relative increase over southern Europe and a relative decrease over the United Kingdom and Scandinavia. All of these features are quantitatively in agreement with those inferred from across-model regressions when the uncertainty on these regressions is taken into account, corroborating the magnitude and structure of the stratospheric influence on tropospheric climate change identified in M2014.

We have quantified the magnitude of this stratospheric influence relative to the CMIP5 model spread, where "spread" here is defined as the CMIP5 $4 \sigma$ range, in two ways: 1) by assessing how much the CMIP5 model spread is reduced once this stratospheric influence has been regressed out and 2) by comparing the influence, in our experiments, of vortex states on opposite sides of the CMIP5 range, with the CMIP5 spread. Using measure 1, it is found that the CMIP5 spread in $\bar{u}$ is reduced by around $10 \%$ at about $60^{\circ} \mathrm{N}$ and about $35^{\circ} \mathrm{N}$ and the spread in $700-\mathrm{hPa}$ $u$ in the North Atlantic is reduced by between 5\% and 10\% upon regressing out the stratospheric contribution. In terms of the SLP response over the Arctic, the spread is reduced by up to $15 \%$, and for precipitation it is reduced by, at most, between $5 \%$ and $10 \%$ over regions of southern Europe, the United Kingdom, and Scandinavia. Through measure 2, that is, assessing the magnitude, relative to the overall CMIP5 spread, of the difference between simulations with vortex states on opposite ends of the CMIP5 range, we find that the difference between an extreme weakening and extreme strengthening of the polar vortex is around $20 \%$ of the spread in high-latitude zonal-mean zonal wind and around $10 \%-15 \%$ farther south; around $20 \%-25 \%$ of the CMIP5 spread in lower-tropospheric zonal wind over the eastern Atlantic and Europe; up to around $50 \%$ of the CMIP5 spread in SLP response over the Arctic; and over southern Europe, the United Kingdom, and Scandinavia, the difference induced by vortex states on the extreme weakening and strengthening edges of the distribution can reach up to about $20 \%$ of the model spread in future precipitation changes.

Overall, uncertainties in the future changes of the stratospheric polar vortex represent a small, but nonnegligible source of uncertainty in tropospheric climate change, particularly for the Arctic and Atlantic and $\mathrm{Eu}-$ ropean sectors. Of considerable impact is the influence on precipitation over Europe. The precipitation anomaly induced by polar vortex changes on opposite ends of the CMIP5 distribution can reach up to $0.25 \mathrm{~mm} \mathrm{day}^{-1}$ over southern Europe and the Mediterranean countries, which 
is equivalent to roughly $10 \%-20 \%$ of their present-day wintertime precipitation climatology (Seager et al. 2014a, their Fig. 1a). For countries in this region that are likely to become increasingly water-stressed in the future, this is a big difference. If the predictions of greater strengthening of the polar vortex in the future are the correct ones, then we might expect the impacts of climate change in these regions to be considerably more severe than predicted by the multimodel mean. At present there is no reason to believe this outcome is more likely than the alternatives; that is, the vortex weakening or remaining more or less unchanged, but it is still a plausible way in which the stratosphere may change in the future. While, in the presence of other sources of uncertainty and internal variability, this is a relatively small contribution to the spread in model predictions, it may be an important one, and a tractable one to reduce through improved understanding, in contrast to the irreducible uncertainty associated with internal variability. This further motivates an improved understanding of the reasons behind the wide spread in stratospheric polar vortex responses among models and an improved constraint on which projections are most likely to occur in the real world.

Acknowledgments. The National Center for Atmospheric Research is sponsored by the National Science Foundation. This work was also funded by National Science Foundation Awards AGS-1317469 and AGS1734760. We are grateful to Yaga Richter for providing the 46-level model and the free-running $1 \times \mathrm{CO}_{2}$ simulation used in this study, and Naftali Cohen for initial analysis that lead to an earlier version of Fig. 1. We acknowledge the World Climate Research Programme's Working Group on Coupled Modelling, which is responsible for CMIP, and we thank the climate modeling groups (listed in Table 1 of this paper) for producing and making available their model output. For CMIP, the U.S. Department of Energy's Program for Climate Model Diagnosis and Intercomparison provides coordinating support and led development of software infrastructure in partnership with the Global Organization for Earth System Science Portals. We would also like to acknowledge high-performance computing support from Yellowstone provided by NCAR's Computational and Information Systems Laboratory, sponsored by the National Science Foundation. This is LDEO contribution number 8226 .

\section{APPENDIX}

\section{Stratospheric Perturbation Design}

For the STRONG4x and WEAK4x experiments, the latitude-pressure structure of the perturbations $\beta_{u}, \beta_{v}$, and $\beta_{T}$ [see (2) and (3)], are derived from the regression, across the 35 CMIP5 models (Table 1), of DJF-averaged future - past difference fields onto $\bar{u}_{\mathrm{pv}}$. For example, for the case of $\bar{u}$, the regression takes the form

$$
\begin{aligned}
\bar{u}(\phi, p, \text { model })= & \alpha_{u}(\phi, p)+\beta_{u}(\phi, p) \bar{u}_{\mathrm{pv}}(\text { model }) \\
& +\varepsilon_{u}(\text { model })
\end{aligned}
$$

where $\alpha_{u}$ and $\beta_{u}$ are the regression coefficients, and $\varepsilon_{u}$ is the residual. Analogous regressions for $\bar{T}$ and $\bar{v}$ are used to obtain the regression coefficients $\beta_{T}$ and $\beta_{v}$. The component related to the globally averaged surface temperature increase is first regressed out from $\bar{u}, \bar{v}$, and $\bar{T}$, but this has very minimal impact on the structures of $\beta_{u}, \beta_{v}$, and $\beta_{T}$.

The regression coefficients $\beta_{u}, \beta_{T}$, and $\beta_{v}$ (Figs. A1a-c) are the latitude-pressure structures for the perturbations below $10 \mathrm{hPa}$. These coefficients show that models with more positive (negative) $\bar{u}_{\mathrm{pv}}$ indices exhibit a coherent strengthening (weakening) of the polar vortex throughout the depth of the stratosphere, with oppositely signed $\bar{u}$ anomalies farther south and a cooling (warming) over the polar cap, along with minimal $\bar{v}$ anomalies. This is very reminiscent of the dominant mode of variability in stratospheric zonal winds on daily time scales (not shown). Note that we retain the anomalies that arise at all latitudes from the CMIP5 regression, not just those in the polar vortex region, but they are relatively small elsewhere.

Since the CMIP5 data are only generally available up to $10 \mathrm{hPa}$, the latitude-pressure structures have to somehow be extended to the model top. We determine the structure above $10 \mathrm{hPa}$ using a smaller subset of eight models (Table 1) for which native model-level data were available and for which the model top was above that of CESML46. These model-level data were first interpolated onto CESML46 levels. The regression coefficients $\beta_{u, 8}$ and $\beta_{T, 8}$ for this eight-model subset (Figs. A1d,e) are similar to those for the 35-model regression (cf. with Figs. A1a,b), although $\beta_{u, 8}$ is lacking the oppositely signed anomaly south of $40^{\circ} \mathrm{N}$. Nevertheless, the structure is sufficiently similar that it can be used to determine an appropriate form for the vertical structure of the perturbations above $10 \mathrm{hPa}$. We base the vertical structure on the structure of $\beta_{T, 8}$ at $80^{\circ} \mathrm{N}$. Poleward of $20^{\circ} \mathrm{N}, \beta_{T}$ of the 35 -model regression is extended above by scaling the $10-\mathrm{hPa}$ values by the following vertical structure factor:

$$
F(p)=\frac{\beta_{T, 8}\left(80^{\circ} \mathrm{N}, p\right)}{\beta_{T, 8}\left(80^{\circ} \mathrm{N}, 10 \mathrm{hPa}\right)}
$$

[Fig. A1f; i.e., $\beta_{T}(\phi, p)=\beta_{T}(\phi, 10 \mathrm{hPa}) F(p)$ for $p<$ $10 \mathrm{hPa}$. South of $20^{\circ} \mathrm{N}$, the $\bar{T}$ anomalies are linearly 
(a) U, 35 models

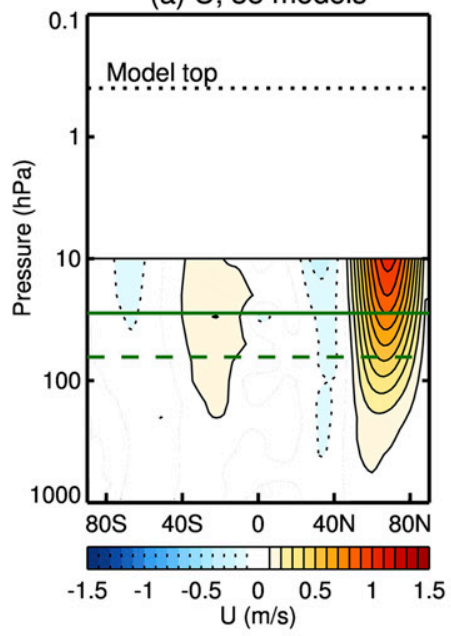

(d) U, 8 models

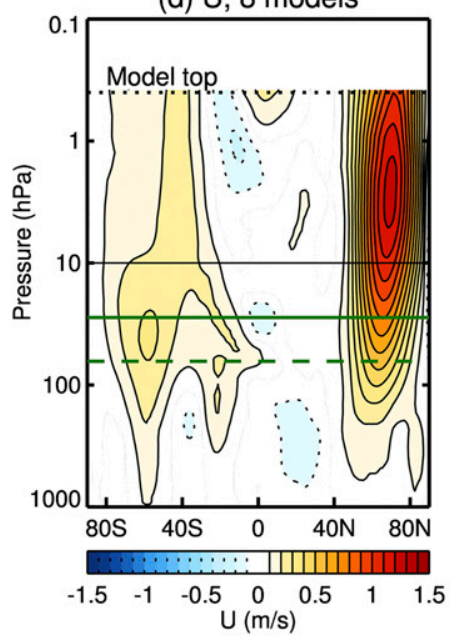

(g) U perturbation

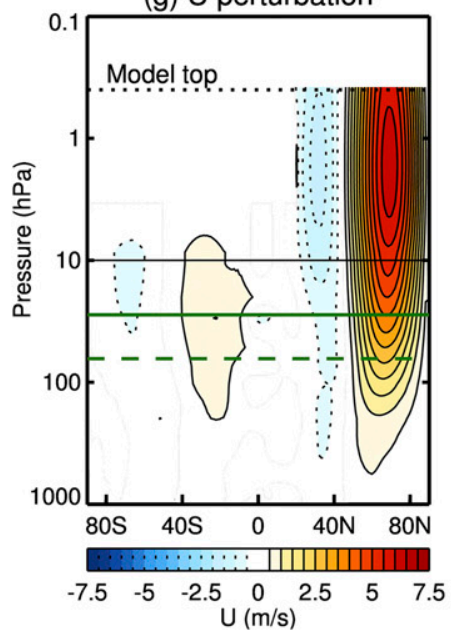

(b) T, 35 models

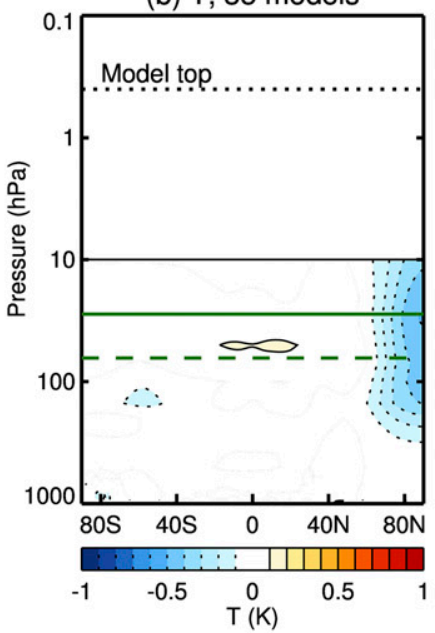

(e) T, 8 models

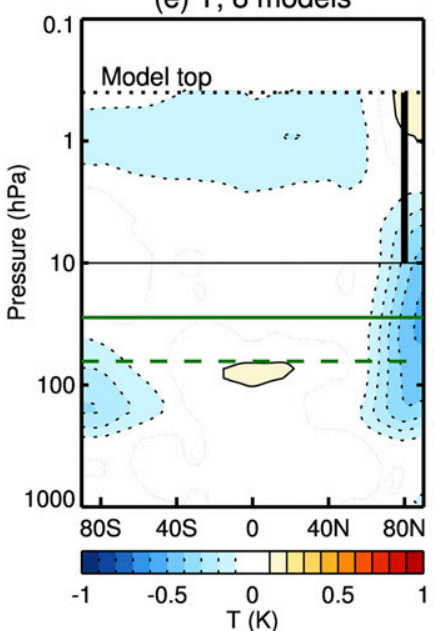

(h) T perturbation

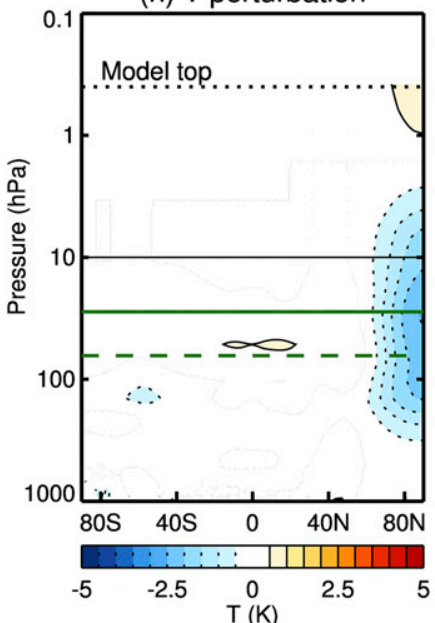

(c) V, 35 models

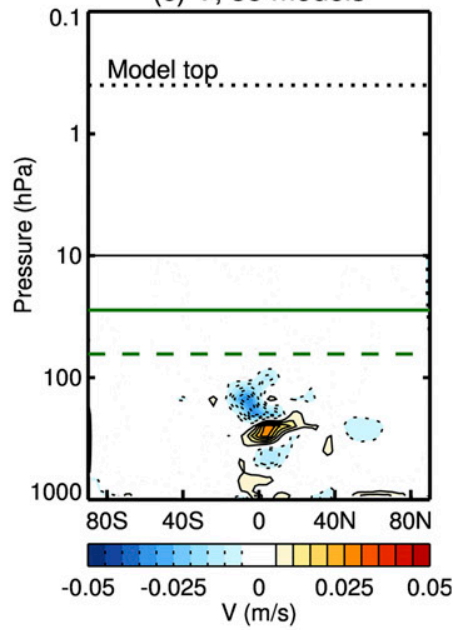

(f) Vertical Structure Factor

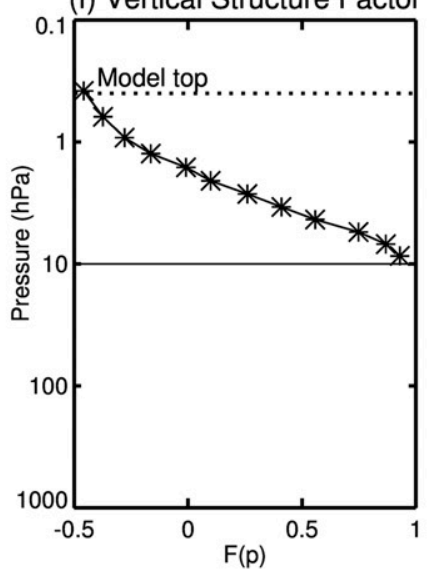

(i) $\mathrm{V}$ perturbation

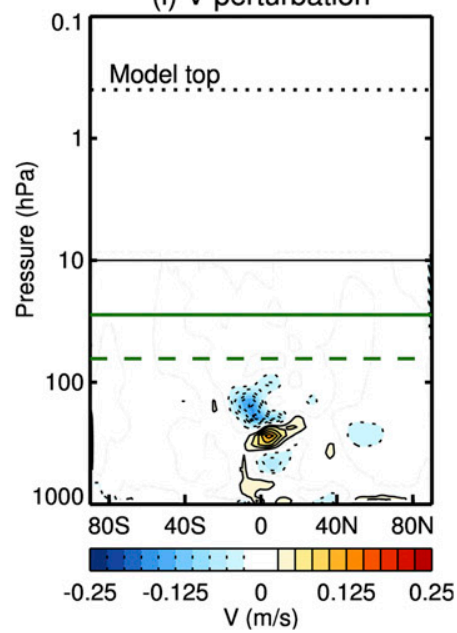

FIG. A1. The linear regression of (a) $\bar{u}$, (b) $\bar{T}$, and (c) $\bar{v}$ onto the $\bar{u}_{\mathrm{pv}}$ index across all 35 CMIP5 models. The linear regression of (d) $\bar{u}$ and (e) $\bar{T}$ onto the $\bar{u}_{\mathrm{pv}}$ index across a subset of eight models that provided model-level data and for which the model top was above $0.4 \mathrm{hPa}$. (f) The vertical structure factor $F(p)$ [(A2)], which is defined using the $\bar{T}$ structure in (e) at $80^{\circ} \mathrm{N}$ [thick solid black vertical line in (e)]. The overall (g) $\bar{u}$, (h) $\bar{T}$, and (i) $\bar{v}$ perturbations constructed by the method outlined in the appendix. The dashed green line shows the level below which zero nudging is applied, and the solid green line shows the level above which full nudging is applied. The horizontal black line shows the 10-hPa level (i.e., the highest level for which CMIP5 data are available for all models). 
(a) $10 \mathrm{hPa} U$ regressed onto $U_{\mathrm{pv}}$

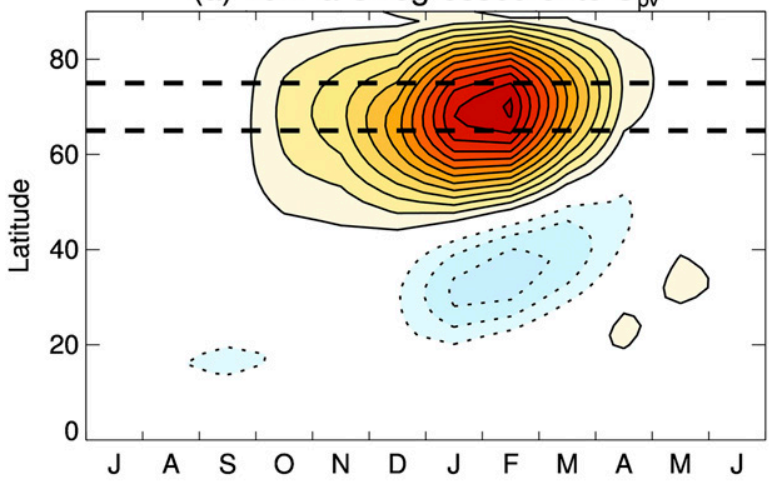

(b) Idealized seasonality $\mathrm{S}(\mathrm{t})$

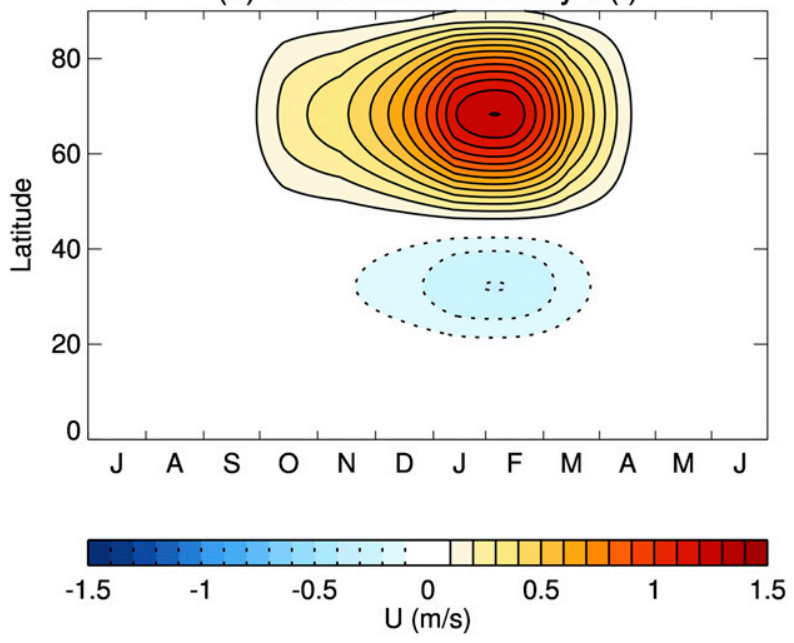

FIG. A2. (a) Monthly variation of future - past difference in 10-hPa zonal-mean zonal wind regressed (across 35 models) onto the DJF $\bar{u}_{\mathrm{pv}}$ index. (b) The idealized perturbations imposed in the model as given by $S(t)$ multiplied by the latitude-pressure structure of Fig. A1g.

tapered to zero between 10 and $3 \mathrm{hPa}$. The $\bar{u}$ anomalies are then those that are in thermal wind balance with this $\bar{T}$ structure according to

$$
\begin{aligned}
\beta_{u}(\phi, p)= & \beta_{u}(\phi, 10 \mathrm{hPa}) \\
& +\int_{\ln (10 \mathrm{hPa})}^{\ln p} \frac{R \partial \beta_{T}(\phi, p)}{\partial \phi} d(\ln p) \text { for } p<10 \mathrm{hPa},
\end{aligned}
$$

and the $\bar{v}$ anomalies are set to zero above $10 \mathrm{hPa}$. This gives the overall latitude-pressure structures shown in Figs. A1g-i. Here, the anomalies have been scaled by 5 , which is the magnitude chosen to span the model spread [see (2) and (3)].

The latitude-pressure structure of the perturbations is based on the DJF-averaged anomalies, but we also give the perturbations an idealized seasonality $S(t)$, intended to mimic the seasonality seen in the CMIP5 model regressions onto $\bar{u}_{\mathrm{pv}}$. Figure A2a shows the monthly regression of $10-\mathrm{hPa}$ zonal winds onto the DJF-averaged $\bar{u}_{\mathrm{pv}}$ index. The anomalous zonal winds associated with DJF-averaged $\bar{u}_{\mathrm{pv}}$ start around October, increase to a maximum in January and February, and subsequently decline through March and April. The seasonality $S(t)$ is given by the quadratic interpolation of the monthly $\bar{u}$ shown in Fig. A2a averaged between $60^{\circ}$ and $75^{\circ} \mathrm{N}$ (i.e., the same latitudes as used for $\bar{u}_{\text {pv }}$ ) onto 6-hourly intervals, which will have a DJF average of approximately 1 by construction. This same seasonality is applied at all latitudes and pressures, giving, for example, at $10 \mathrm{hPa}$, the seasonality shown in Fig. A2b. Therefore, the stratospheric perturbations imposed in STRONG4x and WEAK $4 x$ have the seasonality given by Fig. A2 and do not only consist of anomalies in DJF.

\section{REFERENCES}

Ayarzagüena, B., and Coauthors, 2018: No robust evidence of future changes in major stratospheric sudden warmings: A multi-model assessment from CCMI. Atmos. Chem. Phys. Discuss., https://doi.org/10.5194/acp-2018-296.

Baldwin, M. P., and T. J. Dunkerton, 2001: Stratospheric harbingers of anomalous weather regimes. Science, 294, 581-584, https://doi.org/10.1126/science.1063315.

Barnes, E. A., and D. L. Hartmann, 2010: Testing a theory for the effect of latitude on the persistence of eddy-driven jets using CMIP3 simulations. Geophys. Res. Lett., 37, L15801, https:// doi.org/10.1029/2010GL044144.

, and L. M. Polvani, 2013: Response of the midlatitude jets and of their variability to increased greenhouse gases in the CMIP5 models. J. Climate, 26, 7117-7135, https://doi.org/ 10.1175/JCLI-D-12-00536.1.

— and J. A. Screen, 2015: The impact of Arctic warming on the midlatitude jet stream: Can it? Has it? Will it? Wiley Interdiscip. Rev. Climate Change, 6, 277-286, https://doi.org/ 10.1002/wcc.337.

Bell, C. J., L. J. Gray, and J. Kettleborough, 2010: Changes in Northern Hemisphere stratospheric variability under increasing $\mathrm{CO}_{2}$ concentrations. Quart. J. Roy. Meteor. Soc., 136, 1181-1190, https://doi.org/10.1002/qj.633.

Box, J. E., and Coauthors, 2013: Greenland ice sheet mass balance reconstruction. Part 1: Net snow accumulation (16002009). J. Climate, 26, 3919-3934, https://doi.org/10.1175/ JCLI-D-12-00373.1.

Butchart, N., and Coauthors, 2010: Chemistry-climate model simulations of twenty-first century stratospheric climate and circulation changes. J. Climate, 23, 5349-5374, https://doi.org/ 10.1175/2010JCLI3404.1.

Butler, A. H., D. W. J. Thompson, and R. Heikes, 2010: The steadystate atmospheric circulation response to climate change-like thermal forcings in a simple general circulation model. J. Climate, 23, 3474-3496, https://doi.org/10.1175/2010JCLI3228.1.

Ceppi, P., and D. L. Hartmann, 2016: Clouds and the atmospheric circulation response to warming. J. Climate, 29, 783-799, https://doi.org/10.1175/JCLI-D-15-0394.1. 
_, and T. G. Shepherd, 2017: Contributions of climate feedbacks to changes in atmospheric circulation. J. Climate, 30, 90979118, https://doi.org/10.1175/JCLI-D-17-0189.1.

Chang, E. K. M., Y. Guo, and X. Xia, 2012: CMIP5 multimodel ensemble projection of storm track change under global warming. J. Geophys. Res., 117, D23118, https://doi.org/10.1029/ 2012JD018578.

Charlton-Perez, A. J., and Coauthors, 2013: On the lack of stratospheric dynamical variability in low-top versions of the CMIP5 models. J. Geophys. Res. Atmos., 118, 2494-2505, https://doi.org/10.1002/jgrd.50125.

Delcambre, S. C., D. J. Lorenz, D. J. Vimont, and J. E. Martin, 2013: Diagnosing Northern Hemisphere jet portrayal in 17 CMIP3 global climate models: Twenty-first century projections. J. Climate, 26, 4930-4946, https://doi.org/10.1175/JCLI-D-12-00359.1.

Gerber, E. P., and Coauthors, 2012: Assessing and understanding the impact of stratospheric dynamics and variability on the Earth system. Bull. Amer. Meteor. Soc., 93, 845-859, https:// doi.org/10.1175/BAMS-D-11-00145.1.

Gillett, N. P., M. R. Allen, and K. D. Williams, 2002: The role of stratospheric resolution in simulating the Arctic Oscillation response to greenhouse gases. Geophys. Res. Lett., 29, 1500, https://doi.org/10.1029/2001GL014444.

Harvey, B. J., L. C. Shaffrey, T. J. Woollings, G. Zappa, and K. I. Hodges, 2012: How large are projected 21st century storm track changes? Geophys. Res. Lett., 39, L18707, https://doi.org/ 10.1029/2012GL052873.

,$- \ldots$, and,- 2014 : Equator-to-pole temperature differences and the extra-tropical storm track responses of the CMIP5 climate models. Climate Dyn., 43, 1171-1182, https:// doi.org/10.1007/s00382-013-1883-9.

Hawkins, E., and R. Sutton, 2009: The potential to narrow uncertainty in regional climate predictions. Bull. Amer. Meteor. Soc., 90, 1095-1107, https://doi.org/10.1175/2009BAMS2607.1.

Haynes, P. H., C. J. Marks, M. E. McIntyre, T. G. Shepherd, and K. P. Shine, 1991: On the "downward control" of extratropical diabatic circulations by eddy-induced mean zonal forces. J. Atmos. Sci., 48, 651-678, https://doi.org/10.1175/1520-0469(1991)048<0651: OTCOED $>2.0 . \mathrm{CO} ; 2$.

Hitchcock, P., and P. H. Haynes, 2014: Zonally symmetric adjustment in the presence of artificial relaxation. J. Atmos. Sci., 71, 4349-4368, https://doi.org/10.1175/JAS-D-14-0013.1.

—, and I. R. Simpson, 2014: The downward influence of stratospheric sudden warmings. J. Atmos. Sci., 71, 3856-3876, https://doi.org/10.1175/JAS-D-14-0012.1.

Joseph, R., M. Ting, and P. J. Kushner, 2004: The global stationary wave response to climate change in a coupled GCM. J. Climate, 17, 540-556, https://doi.org/10.1175/1520-0442(2004)017<0540: TGSWRT $>2.0 . \mathrm{CO} ; 2$

Karpechko, A. Y., and E. Manzini, 2012: Stratospheric influence on tropospheric climate change in the Northern Hemisphere. J. Geophys. Res., 117, D05133, https://doi.org/10.1029/2011JD017036.

— , and —, 2017: Arctic stratosphere dynamical response to global warming. J. Climate, 30, 7071-7086, https://doi.org/ 10.1175/JCLI-D-16-0781.1.

Kidston, J., and E. P. Gerber, 2010: Intermodel variability of the poleward shift of the austral jet stream in the CMIP3 integrations linked to biases in 20th century climatology. Geophys. Res. Lett., 37, L09708, https://doi.org/10.1029/2010GL042873.

Kushner, P. J., and L. M. Polvani, 2004: Stratosphere-troposphere coupling in a relatively simple AGCM: The role of eddies. J. Climate, 17, 629-639, https://doi.org/10.1175/1520-0442(2004)017<0629: SCIARS $>2.0 . \mathrm{CO} ; 2$.
Lorenz, D. J., and E. T. DeWeaver, 2007: Tropopause height and zonal wind response to global warming in the IPCC scenario integrations. J. Geophys. Res., 112, D10119, https://doi.org/ 10.1029/2006JD008087.

Manzini, E., and Coauthors, 2014: Northern winter climate change: Assessment of uncertainty in CMIP5 projections related to stratosphere-troposphere coupling. J. Geophys. Res. Atmos., 119, 7979-7998, https://doi.org/10.1002/ 2013JD021403.

McLandress, C., and T. G. Shepherd, 2009: Impact of climate change on stratospheric sudden warmings as simulated by the Canadian Middle Atmosphere Model. J. Climate, 22, 54495463, https://doi.org/10.1175/2009JCLI3069.1.

Mitchell, D. M., S. N. Osprey, L. J. Gray, N. Butchart, S. C Hardiman, A. J. Charlton-Perez, and P. Watson, 2012: The effect of climate change on the variability of the Northern Hemisphere stratospheric polar vortex. J. Atmos. Sci., 69, 2608-2618, https://doi.org/10.1175/JAS-D-12-021.1.

Neelin, J. D., B. Langenbrunner, J. E. Meyerson, A. Hall, and N. Berg, 2013: California winter precipitation change under global warming in the Coupled Model Intercomparison Project phase 5 ensemble. J. Climate, 26, 6238-6256, https://doi. org/10.1175/JCLI-D-12-00514.1.

Perlwitz, J., and N. Harnik, 2003: Observational evidence of a stratospheric influence on the troposphere by planetary wave reflection. J. Climate, 16, 3011-3026, https://doi.org/10.1175/ 1520-0442(2003)016<3011:OEOASI $>2.0$. CO;2.

Polvani, L. M., L. Sun, A. H. Butler, J. H. Richter, and C. Deser, 2017: Distinguishing stratospheric sudden warmings from ENSO as key drivers of wintertime climate variability over the North Atlantic and Eurasia. J. Climate, 30, 1959-1969, https:// doi.org/10.1175/JCLI-D-16-0277.1.

Richter, J. H., F. Sassi, and R. R. Garcia, 2010: Toward a physically based gravity wave source parameterization in a general circulation model. J. Atmos. Sci., 67, 136-156, https://doi.org/ 10.1175/2009JAS3112.1.

_ C. Deser, and L. Sun, 2015: Effects of stratospheric variability on El Niño teleconnections. Environ. Res. Lett., 10, 124021, https://doi.org/10.1088/1748-9326/10/12/124021.

Rind, D., D. Shindell, P. Lonergan, and N. K. Balachandran, 1998: Climate change and the middle atmosphere. Part 3: The doubled $\mathrm{CO}_{2}$ climate revisited. J. Climate, 11, 876-894, https://doi.org/ 10.1175/1520-0442(1998)011<0876:CCATMA > 2.0.CO;2.

Scaife, A. A., and Coauthors, 2012: Climate change projections and stratosphere-troposphere interaction. Climate Dyn., 38, 2089 2098, https://doi.org/10.1007/s00382-011-1080-7.

Seager, R., H. Liu, N. Henderson, I. Simpson, C. Kelley, T. Shaw, Y. Kushnir, and M. Ting, 2014a: Causes of increasing aridification of the Mediterranean region in response to rising greenhouse gases. J. Climate, 27, 4655-4676, https://doi.org/ 10.1175/JCLI-D-13-00446.1.

_ , and Coauthors, 2014b: Dynamical and thermodynamical causes of large-scale changes in the hydrological cycle over North America in response to global warming. J. Climate, 27, 7921-7948, https://doi.org/10.1175/JCLI-D-14-00153.1.

Shaw, T. A., J. Pelwitz, and N. Harnik, 2010: Downward wave coupling between the stratosphere and troposphere: The importance of meridional wave guiding and comparison with zonal-mean coupling. J. Climate, 23, 6365-6381, https://doi.org/ 10.1175/2010JCLI3804.1.

, and Coauthors, 2016: Storm track processes and the opposing influences of climate change. Nat. Geosci., 9, 656-664, https:// doi.org/10.1038/ngeo2783. 
Shindell, D. T., R. L. Miller, G. A. Schmidt, and L. Pandolfo, 1999: Simulation of recent northern winter climate trends by greenhouse-gas forcing. Nature, 399, 452-455, https://doi.org/ $10.1038 / 20905$

Sigmond, M., and J. F. Scinocca, 2010: The influence of the basic state on the Northern Hemisphere circulation response to climate change. J. Climate, 23, 1434-1446, https://doi.org/ 10.1175/2009JCLI3167.1.

- - _ - and P. J. Kusher, 2008: Impact of the stratosphere on tropospheric climate change. Geophys. Res. Lett., 35, L12706, https://doi.org/10.1029/2008GL033573.

Simpson, I. R., and L. M. Polvani, 2016: Revisiting the relationship between jet position, forced response, and annular mode variability in the southern midlatitudes. Geophys. Res. Lett. 43, 2896-2903, https://doi.org/10.1002/2016GL067989.

— - M. Blackburn, and J. D. Haigh, 2009: The role of eddies in driving the tropospheric response to stratospheric heating perturbations. J. Atmos. Sci., 66, 1347-1365, https://doi.org/ 10.1175/2008JAS2758.1.

—, P. Hitchcock, T. G. Shepherd, and J. F. Scinocca, 2011: Stratospheric variability and tropospheric annular-mode timescales. Geophys. Res. Lett., 38, L20806, https://doi.org/ 10.1029/2011GL049304.

- T. A. Shaw, and R. Seager, 2014: A diagnosis of the seasonally and longitudinally varying midlatitude circulation response to global warming. J. Atmos. Sci., 71, 2489-2515, https://doi.org/10.1175/JAS-D-13-0325.1.

Song, Y., and W. A. Robinson, 2004: Dynamical mechanisms for stratospheric influences on the troposphere. J. Atmos. Sci., 61 1711-1725, https://doi.org/10.1175/1520-0469(2004)061<1711 DMFSIO $>2.0 . \mathrm{CO} ; 2$.

Stephenson, D. B., and I. M. Held, 1993: GCM response of northern winter stationary waves and storm tracks to increasing amounts of carbon dioxide. J. Climate, 6, 1859-1870, https://doi.org/ 10.1175/1520-0442(1993)006<1859:GRONWS>2.0.CO;2.
Swart, N. C., and J. C. Fyfe, 2012: Observed and simulated changes in the Southern Hemisphere surface westerly wind-stress. Geophys. Res. Lett., 39, L16711, https://doi.org/10.1029/ 2012GL052810

Taylor, K. E., R. J. Stouffer, and G. A. Meehl, 2012: An overview of CMIP5 and the experiment design. Bull. Amer. Meteor. Soc., 93, 485-498, https://doi.org/10.1175/BAMS-D-11-00094.1.

Voigt, A., and T. A. Shaw, 2015: Circulation response to warming shaped by radiative changes of clouds and water vapour. Nat. Geosci., 8, 102-106, https://doi.org/10.1038/ngeo2345.

Wenzel, S., V. Eyring, E. P. Gerber, and A. Y. Karpechko, 2016: Constraining future summer austral jet stream positions in the CMIP5 ensemble by process-oriented multiple diagnostics regression. J. Climate, 29, 673-687, https://doi.org/10.1175/ JCLI-D-15-0412.1.

Wilcox, L. J., A. J. Charlton-Perez, and L. J. Gray, 2012: Trends in austral jet position in ensembles of high- and low-top CMIP5 models. J. Geophys. Res., 117, D13115, https://doi.org/10.1029/ 2012JD017597.

Wittman, M. A. H., A. J. Charlton, and L. M. Polvani, 2007: The effect of lower stratospheric shear on baroclinic instability. J. Atmos. Sci., 64, 479-496, https://doi.org/10.1175/ JAS3828.1.

Woollings, T., and M. Blackburn, 2012: The North Atlantic jet stream under climate change and its relation to the NAO and EA patterns. J. Climate, 25, 886-902, https://doi.org/10.1175/ JCLI-D-11-00087.1.

Yin, J. H., 2005: A consistent poleward shift of the storm tracks in simulations of 21 st century climate. Geophys. Res. Lett., 32, L18701, https://doi.org/10.1029/2005GL023684.

Zappa, G., B. J. Hoskins, and T. G. Shepherd, 2015: Improving climate change detection through optimal seasonal averaging: The case of the North Atlantic jet and European precipitation. J. Climate, 28, 6381-6397, https://doi.org/10.1175/ JCLI-D-14-00823.1. 